Illinois State University

ISU ReD: Research and eData

Theses and Dissertations

$6-29-2020$

\title{
Rapid Vertical Flow Assay On Aunp Plasmonic Paper For Sers- Based Point Of Need Diagnostics
}

Richard Frimpong

Illinois State University, rkfrimpong@yahoo.com

Follow this and additional works at: https://ir.library.illinoisstate.edu/etd

Part of the Chemistry Commons

\section{Recommended Citation}

Frimpong, Richard, "Rapid Vertical Flow Assay On Aunp Plasmonic Paper For Sers-Based Point Of Need Diagnostics" (2020). Theses and Dissertations. 1286.

https://ir.library.illinoisstate.edu/etd/1286

This Thesis is brought to you for free and open access by ISU ReD: Research and eData. It has been accepted for inclusion in Theses and Dissertations by an authorized administrator of ISU ReD: Research and eData. For more information, please contact ISUReD@ilstu.edu. 


\section{RAPID VERTICAL FLOW ASSAY ON AUNP PLASMONIC PAPER FOR SERS-BASED POINT OF NEED DIAGNOSTICS}

\section{RICHARD FRIMPONG}

\section{Pages 72}

SERS based immunoassays for point-of-care diagnostics is a promising tool to facilitate biomarker detection for early disease diagnosis and control. The technique is based on a sandwiched system in which antigen is first captured by a selective substrate and then labeled by an extrinsic Raman label (ERL). Here, we report on the use of gold nanoparticle modified filter paper as a novel capture membrane in a vertical flow format. This vertical flow configuration affords reproducible flow of sample and label through the capture substrate to overcome diffusion limited kinetics and significantly reduced assay time. The filter paper was selected due to its affordability and availability, while the embedded AuNPs maximized plasmonic coupling and SERS enhancement. Additionally, the embedded AuNP served as a scaffold to immobilize capture antibody to specifically bind antigen. In this work, a SERS-based rapid vertical flow (SERS-RVF) immunoassay for detection of mouse IgG was developed to establish proof of principle. Optimization of assay conditions led to a limit of detection of $3 \mathrm{ng} / \mathrm{mL}$, which is comparable to more traditional formats carried out in multi-well plates and significantly reduced assay time to less than 2 minutes. Additionally, IgG was accurately quantified in normal serum to validate the SERS-RVF assay for application to the analysis of biological samples. These results highlight the potential advantages of the SERS-RVF platform for point-of-need testing. 
KEYWORDS: Immunoassay; surface-enhanced Raman scattering (SERS); plasmonic paper; vertical flow assay; point-of-need (PON) testing; point-of-care (POC) diagnostics 


\title{
RAPID VERTICAL FLOW ASSAY ON AUNP PLASMONIC PAPER FOR SERS-BASED POINT OF NEED DIAGNOSTICS
}

\author{
RICHARD FRIMPONG
}

A Thesis Submitted in Partial Fulfillment of the Requirements for the Degree of

MASTER OF SCIENCE

Department of Chemistry ILLINOIS STATE UNIVERSITY 
C 2020 Richard Frimpong 


\title{
RAPID VERTICAL FLOW ASSAY ON AUNP PLASMONIC PAPER FOR SERS-BASED POINT OF NEED DIAGNOSTICS
}

\author{
RICHARD FRIMPONG
}

COMMITTEE MEMBERS:

Jeremy D. Driskell, Chair

Jun-Hyun Kim

Michael Webb 


\section{ACKNOWLEDGMENTS}

My first thanks go to Almighty God for the many blessings he showered on me throughout my graduate studies.

I would like to express my sincere gratitude to my primary supervisor, Dr. Jeremy D. Driskell. He always accommodating and have a helping hand if I had question about my research or writing. He shows support in everything I do and teach me the right direction for my thesis writing. Your support and encouragement were worth more than I can express.

I would also like to give big thank you to Dr. Jun-Hymn Kim for his constant motivation and assistance throughout my graduate school. I really appreciate your valuable comments through the process of researching and writing this thesis it really helps me a lot to improve my thesis writing.

I would also like to express appreciation to ISU chemistry department faculty, staff and my fellow graduate students for their love, unfailing support and assistance.

Finally, I express my gratitude to my parents and siblings for providing me with unfailing support and continuous encouragement throughout my years of study. This accomplishment would not have been possible without them.

R. F 


\section{CONTENTS}

Page

ACKNOWLEDGMENTS

TABLES

FIGURES

CHAPTER I: INTRODUCTION

Significance of Study 1

$\begin{array}{ll}\text { Point-of-Care Testing } & 1\end{array}$

Immunoassay and its Application $\quad 2$

Formats of Immunoassay $\quad 3$

Antibodies and its Structural Activities $\quad 5$

$\begin{array}{ll}\text { Established Immunoassay Formats } & 7\end{array}$

$\begin{array}{ll}\text { Surface Enhanced Raman Spectroscopy (SERS) } & 10\end{array}$

$\begin{array}{ll}\text { SERS Hot Spots } & 12\end{array}$

$\begin{array}{ll}\text { SERS-Based Immunoassay } & 13\end{array}$

$\begin{array}{ll}\text { SERS Substrates } & 16\end{array}$

$\begin{array}{ll}\text { Paper-Based Assay (PBA) } & 18\end{array}$

$\begin{array}{ll}\text { Thesis Objectives } & 18\end{array}$

CHAPTER II: MATERIALS AND METHODS 20

$\begin{array}{ll}\text { Materials and Reagents } & 20\end{array}$

$\begin{array}{ll}\text { Synthesis of Gold Nanoparticles (AuNPs) } & 20\end{array}$

Preparation of Plasmonic Paper Capture Substrate $\quad 21$

Preparation of Extrinsic Raman Labels (ERLs) $\quad 21$ 
Vertical Flow Immunoassay Protocol

Characterization Methods

Scanning Electron Microscope (SEM)

Surface Enhanced Raman Spectroscopy (SERS)

Dynamic Light Scattering (DLS)

UV-Visible Spectrometer

CHAPTER III: RESULTS AND DISCUSSION

Assay Design and Sensing Principle

Fabrication and Characterization of Plasmonic Paper

Role of Plasmonic Paper

Optimization of Assay Protocol

Amount of capture antibody

Volume of extrinsic Raman labels (ERLs)

Volume of rinsing buffer

Analytical Performance -Dynamic Range, Detection Limit, Reproducibility, Selectivity

Application of the SERS-VFA to the Analysis of Mouse Serum

Research Summary

Section A: Optimization of Assay Parameters

Section B: Analytical Performance of the SERS-VFA

Section C: Application of the SERS-VFA to Determine IgG in Mouse Serum

Future Works 


\section{TABLES}

Table

Page

1. Mouse $\operatorname{IgG}(\mathrm{mIgG})$ Diluted in PBS

2. Average SERS intensity of diluted mouse serum 


\section{FIGURES}

Figure

1. Immunoassay formats (a) Non-competitive (b) Response of non-competitive 5

2. $\quad$ Structure of immunoglobin $\mathrm{G}(\mathrm{IgG}) \quad 7$

3. Principle of enzyme linked immunosorbent assay 9

4. Principle of lateral flow assay 10

5. Electromagnetic effect of SERS 12

6. Illustration of SERS-hot spot (red) 13

7. Overview of SERS VFA 19

8. Illustration of the SERS-vertical flow immunoassay protocol 26

9. SEM images of plasmonic filter papers with their UV analysis of AuNPs solution of grade number 4 and 40 with their corresponding UV analysis of before and $\begin{array}{ll}\text { after loading on the papers } & 28\end{array}$

10. Surface UV-Vis-IR spectra of AuNPs loaded onto plasmonic papers of grade number 4 and 40 filter papers

11. SERS spectra collected using bare filter paper, plasmonic filter papers of grade 4 filter paper, and grade 40 plasmonic filter paper as the capture substrate in a SERS-RVF immunoassay

12. Effect of the amount of capture antibody on plasmonic filter paper grade number 4

13. Effect of ERL volume on SERS intensity of plasmonic papers grade 4 capture substrate

14. Effect of PBS rinsing volume on SERS intensity of capture substrate

15. Illustration of SERS spectra of positive and negative control samples.

16. SERS spectra of concentration dependent assay

17. Calibration curve of the VFA using the optimized conditions with limit of detection

18. Calibration plots for absolute averaged intensity of three independent assays 
19. Calibration plots for normalized averaged intensity of three independent assays 44

20. Calibration plots of mouse IgG prepared in PBS and 10\% rabbit serum 46

21. Calibration curve to determine IgG in mouse serum obtained using optimized condition

22. Limit of linearity of the calibration curve 48

23. SERS spectra of concave cube AuNPs solution 3rd layer analysis of mouse IgG 55 


\section{CHAPTER I: INTRODUCTION}

\section{Significance of Study}

The health and well-being of a nation's labor force determine the long-term social and economic stability of that nation. The world health and technology has seen many improvements the last 50 years [1]. However, there is still more to do as millions die each year in populated countries due to infectious diseases like malaria, AIDS and tuberculosis. These deaths are sometimes due to the challenge of easy treatment options such as in developed countries. Ability to precisely diagnose diseases at their onset of development is a key to determining the origin of the disease. Research proves that the inability to rapidly diagnose diseases which are treatable and preventable at their early stage accounts for the progressive increase in the number of deaths especially in the low- and middle-income countries $[1,2]$.

Recent improvement in the disease diagnosis has been focusing on early detection of disease to efficiently provide good medication in order to control the passing pace of patients. For some time now, many healthcare practitioners have been relying on sophisticated laboratorybased procedures to diagnose disease which has the limitation of long analysis time and requires expertise to generate results. The current advancement and development in the disease diagnosis has been focusing on point-of-care testing (POCT). POCT is the integral part of medicine, it enables simple automated testing that yield fast results.

\section{Point-of-Care Testing}

POCT refers to diagnostic test that is performed in proximity of patients where medical attention is needed to diagnose, monitor and control disease $[3,4]$. The test is typically performed by doctors, laboratory personal or patients. Samples such as blood are collected from 
the patients and analyzed. POCT devices offer advantage of rapid diagnosis of the disease, minimal sample volume for testing portability and cost effectiveness. POCT are normally used in hospitals, paramedic support vehicles and many other environments [5]. The ability of a POCT device to selectively extract and detect the disease biomarker from the patient sample's matrix such as the serum, blood and urine and provide results within a short time makes it an attractive in the diagnostic arena. The demand for POCT devices has been increasing due to the corresponding global increase of chronic and infectious diseases.

Currently, there have been huge investments in the field resulting in a high value of the global POCT market [6], which was estimated at $\$ 13.87$ billion in 2017 and is estimated to grow over $\$ 23.92$ billion by 2026 [7]. Recently, there have been enormous research publications aiming to improve the current POCT. The majority of these publications have been focused on increasing sensitivity, affordability and simplicity of the device. The most common POCT platforms are based on lab-on-a-chip [8], paper [9], microfluidics [10] and novel assay formats $[11,12]$.

\section{Immunoassay and its Application}

The advancement in complementary technologies and the continuous improvements play a vital role in development of next-generation POCT technologies. One key area that is gaining much attention for development of point-of-care testing is the immunoassay, which has been extensively utilized from basic research science to clinical settings, due primarily to the specificity of antibodies for their molecular antigen counterparts.

Immunoassay refers to a test in which responses are based on the interactions between antibody and antigen (analyte) and are commonly relied on for the diagnosis of many infectious 
diseases $[13,14]$. The technique was developed by Rosalyn Sussman Yallow in 1950 which resulted in the Nobel Prize in 1977 [15]. The technique offers several advantages such as inexpensive testing, high sensitivity, high selectivity and can be applied to various targets. The most common applications include drug testing [16], hormone testing [17, 18], bacterial or viral testing $[19,20]$ and environmental testing $[21,22]$.

The main principle of immunoassays is based on the ability of the antibody to recognize and bind to specific antigen to form an antibody-antigen complex also refers to as an immunocomplex. This complex formation is then separated from the unbound antigens througha series of washing steps and are detected by measuring the activity of a label that is covalently link to either antibody or antigen depending on the interest of the assay. Immunoassays have been used in various applications and there have been many research publications to improve the field $[20,21,23,24]$.

\section{Formats of Immunoassay}

Immunoassays come in two main forms: heterogeneous and homogeneous. In the case of heterogeneous, either the antibody or antigen is immobilized on a solid support (for example test tubes, microplates, latex beads, filter paper or magnetic particles) followed by washing to

remove any unbound material [15]. The heterogeneous immunoassay comes in competitive and noncompetitive formats, the noncompetitive format is displayed in Figure 1a [25]. Here, the antigen sample is incubated with an excess of antibody reagent. All the antigen molecules become bound, but not all the antibody binding sites are occupied. To detect the amount of antigen attached to an antibody, a second, labelled antibody is added which binds to another epitope of the antigen. This leads to formation of a sandwich-complex between the primary 
antibody, the sample antigen and the labelled secondary antibody. After washing off any excess reagent, the sandwich-complexes containing the label can be detected and the signal generated is directly related to the amount of antigen in the sample. The response of this type of format is displayed in Figure $1 \mathrm{~b}$ [25]. The labelled secondary antibody binds directly to the target antigenantibody complex. So, the larger the number of sample molecules, the larger the signal, but with very high or low concentration the curve flattens out [30]. Heterogeneous immunoassays are used in most environmental and clinical applications as a result of the complex samples typically encountered. A good example of application of this format is HIV-antibody detection [26, 27] .

In a homogeneous immunoassay format, there are no separation steps of the immunoreagents which sometimes results in matrix interferences.

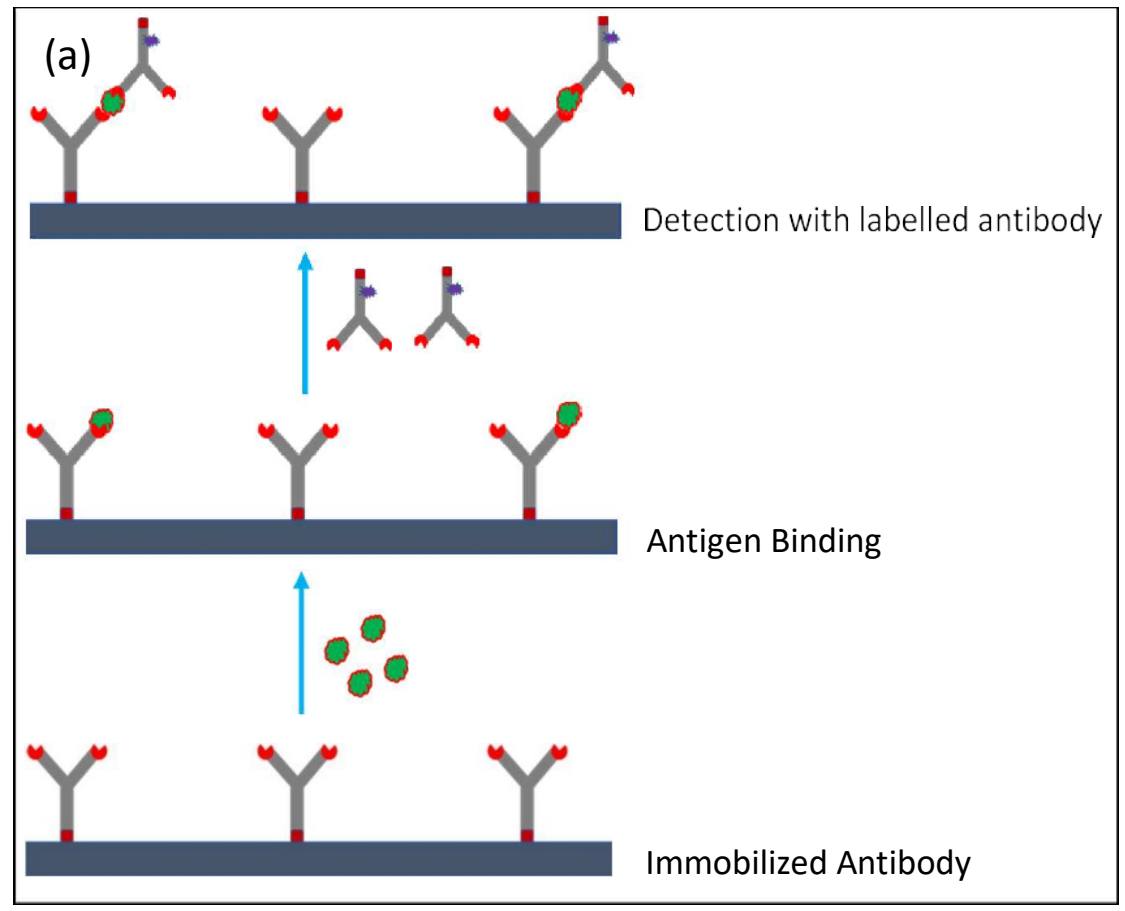




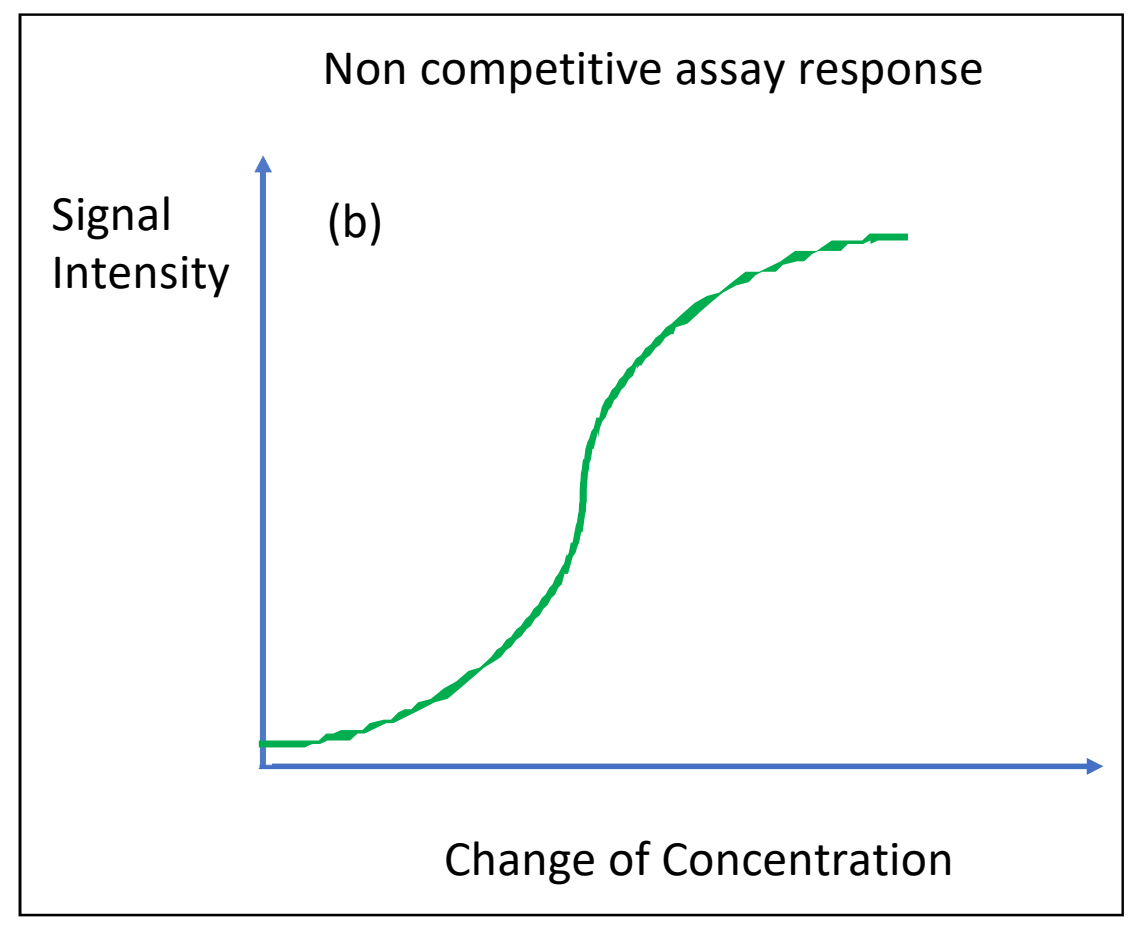

Figure 1. Immunoassay formats (a) Non-competitive (b) Response of non-competitive

\section{Antibodies and its Structural Activities}

Antibodies, also referred to as immunoglobins, are proteins that are essential in the immune system. They recognize and neutralizes pathogens or antigens (Ag) (proteins, polysaccharides, nucleic acids) that invade the body and stimulate the production of immunoglobins. Antibodies have been used in various biosensor platforms including electrochemical [28], fluorescent [29] and colorimetric assays [30]. There are two main types of antibodies that are used in immunoassay, monoclonal or polyclonal. Monoclonal antibodies bind only one single binding site (epitope) on the antigen, while the polyclonal antibodies are able to bind to different sites (epitopes) of the same antigen. The use of monoclonal antibodies has the 
advantage of less cross-reactivity due to its specificity to one binding site than the polyclonal, however, many biosensors make use of polyclonal due to its low cost of production.

Immunoglobin $\mathrm{G}(\mathrm{IgG})$ has a general structure which consists of a distinctive $\mathrm{Y}$-shape and are composed of two heavy chains and two light chains as shown in the Figure 2. These chains are held together by disulfide bonds. The regions that bind to pathogens are located at the tips of the two arms formed by heavy and light chain domain called hypervariable region. The hypervariable region also referred to as $\mathrm{Fab}$ or paratope ends with the $\mathrm{NH}_{3}{ }^{+}$terminal of amino acid that binds to the epitope (binding site of antigens). The Fab of each antibody is unique and allows the antibody to interact with a specific antigen. The antibody and antigen interact in a lock and key manner. This unique feature of the antibody explains the selectivity and specificity of immunoassays and their value for the detection of diseases. There are five types of antibodies found in human and other mammals. Thus $\operatorname{IgG}, \operatorname{IgM}, \operatorname{IgA}, \operatorname{IgD}$ and $\operatorname{IgE}$, the most abundant one is the IgG. The IgG types have a molecular weight of approximately $150 \mathrm{kDa}$. Each type has different shape and size. 


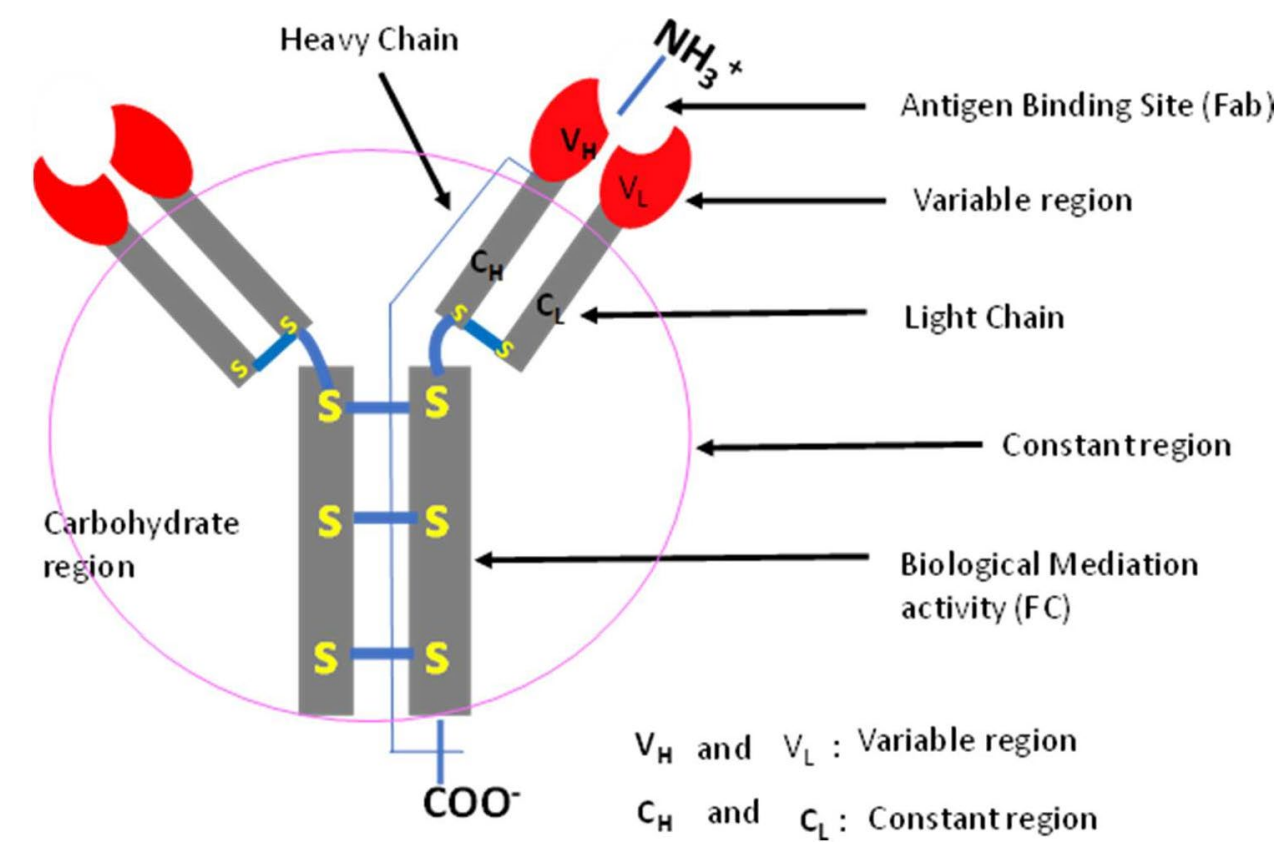

Figure 2. Structure of immunoglobin $\mathrm{G}(\mathrm{IgG})$. Red variable region of light and heavy chain for antigen binding site and grey constant region that has heavy and light chain.

\section{Established Immunoassay Formats}

The complex formation of the immunochemical reaction between the antibody and antigen serve as the basis of the immunoassay. They may be visualized or detected by measuring the activities of the label used. The type of label will determine the format of the immunoassay assay and the method of detection. Several prominent assay formats have been developed during the last two decades for highly prospective POCT. A recent review has provided a summary of the various detection methods with different advantages and limitations [31]. Labels and detection techniques such as fluorescence polarization [32], chemiluminescence [33], electrochemical detection [34] and surface enhanced Raman spectroscopy (SERS) [35], as well as more novel labels, have also been explored to enhance the detection of trace analytes [36]. 
The current method of detection that is employed as standard diagnostic platforms are the enzyme-linked immunosorbent assay (ELISA) [26, 30, 37] and the lateral flow assay (LFA) $[38,39]$. ELISA uses enzyme labels such as alkaline phosphatase and horseradish peroxidase on the secondary antibody providing a colored end product. In one form of ELISA, sample containing the antigen is added to a well plate which is coated with the antigen-specific antibody and then incubated for several hours. After incubation a series of washing is done to remove excess unbound antigens, then an enzyme label antibody is added followed by the addition of substrate which results in the formation of a colored product. The quantity of the antigen present is quantified by measuring the absorbance of the colored product. Figure 3 illustrates the ELISA process. ELISA offers quantitative information but it is a time-consuming multistep process [40]. LFA has also been used as an alternative to reduce turnaround time by providing rapid results for several analyses [41]. LFA makes use of a nitrocellulose membrane test strip which allows samples to flow laterally through the strip by the action of capillary force provided by absorbent pads. Here the sample is applied on a sample pad and begins to flow laterally over the conjugate pad where the analyte binds with the conjugate's particles. Figure 4 details the process of LFA [38]. LFA is rapid and simple technique which is used as a point-of-care diagnostic by healthcare professionals, patients and individuals. LFA has been extensively utilized for various analysis, for example, the most widely used pregnancy tests are based on the LFA principle where the human hormone chorionic gonadotropin (hCG) in urine is detected [42]. LFAs have also been develop for the detection of cancer biomarkers [43], HIV diagnosis [44, 45] and nucleic acid testing $[46,47]$. Despite the importance of this platform, it also comes with several limitations including poor limit of detection [48]. According to reports from Hurt et al [49], LFA was used in population screening of people infected with influenza virus using the commercially 
available products Binax Now Flu A\&B, BD Directigen EZ Flu A+B, Rockedby influenza A and Quidel Quickvalue Flu A+B. The LFAs only identified 73\%, 69\%, 10\% and 67\% respectively of the know positive cases [49]. Recently, rapid vertical flow (RVF) assays have been explored as an alternative to LFAs in an effort to reduce the false negative rates and provide even faster analysis $[50,51]$.

Given these limitations, there has been several reports on ways to improve the uses of these conventional flow assay platforms, with majority of them focusing on enhancing the sensitivity using novel detection modalities such as surface plasmon resonance (SPR) [52], nanowires [53], and quantum dots [54]. However, each of these improvements sacrifices the simplicity, precision and low cost of POCT. An alternative to these detection modalities is SERS. The exceptional properties of SERS such as high sensitivity, potential for multiplexed detection, photostability, and portability allows it to be more useful than other techniques in the diagnostic arena especially with the recent development of portable hand-held Raman spectrometers [55].

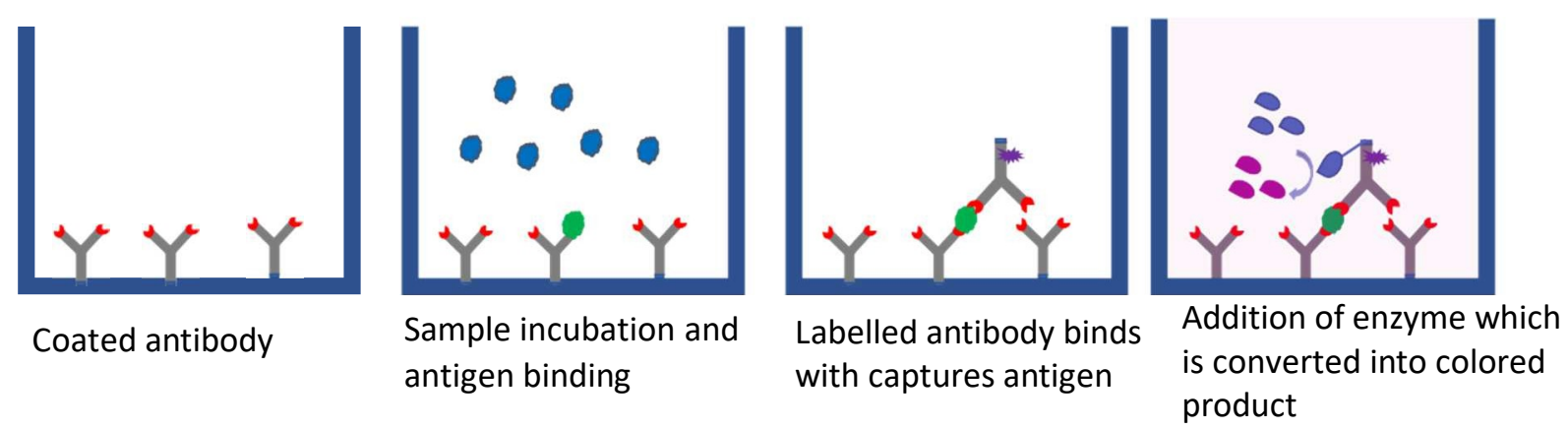

Figure 3. Principle of enzyme linked immunosorbent assay. 
(a)

$$
\text { Sample Conjugate }
$$

(b)

pad pad

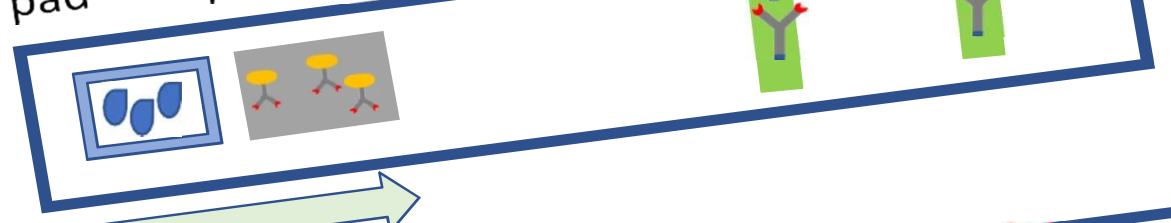

(c)

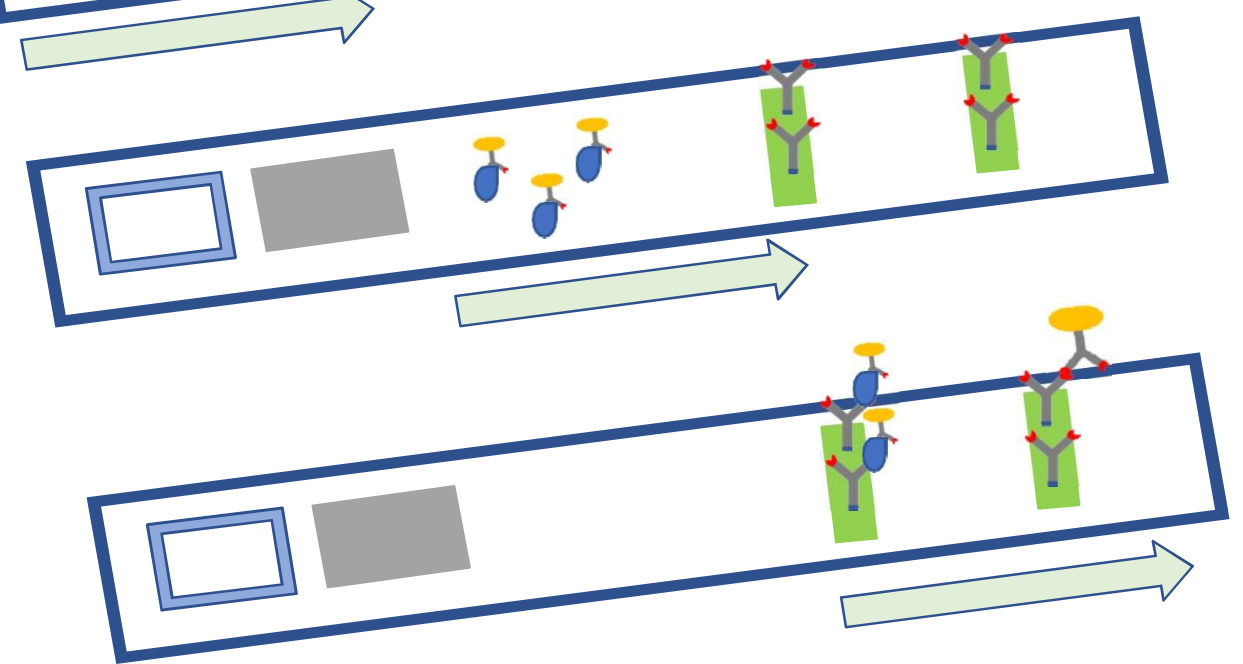

Figure 4. Principle of lateral flow assay. (a) sample is applied to sample pad (b) sample bind with conjugates and flow across by capillary force (c) conjugates are bind to immobilized antibody at the test line to form a color and the control line indicates that.

\section{Surface Enhanced Raman Spectroscopy (SERS)}

Raman spectroscopy is a vibrational spectroscopy resulting from inelastic scattering of a monochromatic light (e.g laser) by atoms of a molecule. Raman spectroscopy is widely used for the analysis of biological materials such as proteins [56], DNA [57] and chromosomes [58]. The molecular composition and the extent of atomic interactions contribute to the vibrational spectrum of the molecule, which provides valuable information for identification and quantification of the molecule. However, a large concentration of the analyte molecule is required to enable the Raman spectroscopic analysis. This has been a major disadvantage to the 
use of Raman spectroscopy for the analysis of biological compounds which are present in small quantities resulting in the generation of weak signal from the spectrum. Moreover, a high laser power of about $300 \mathrm{~mW}$ is required which can cause damage to biological samples.

The limitation of Raman spectroscopy has been overcome through SERS. SERS is defined as an analytical technique that is used to obtain the vibrational spectrum of molecules adsorbed onto metallic nanostructures which provides large SERS active surface area for signal enhancement. SERS has become more useful and has been found in many applications [59] due to its ability to enhance the Raman signal by a factor of $10^{10}[60]$. The SERS phenomenon is a result of the energy changes of the light scattered by vibrating molecules adsorbed on a metallic surface [61]. The factors contributing to SERS enhancement have been found to be chemical and electromagnetic in nature [62].

The electromagnetic (EM) effect has been found to be the strongest contribution to the SERS enhancement due to the strong vibration of the molecules experienced at the surface of the metal particle. In the EM phenomenon, the electromagnetic wave of an incident laser impinges at the metal and dielectric interfaces which energizes the delocalized conduction electrons of the metal nanostructures into collective oscillation (Figure 5). When the frequency of the incident light matches with the inherent oscillation frequency of free electrons in the metal, SPR occurs. The magnitude of the SPR generated depends on the size, shape, electron density and dielectric environment of the nanostructure [63]. The SPR that is generated is highly localized on the metallic surface and is referred to as localized surface plasmon resonance (LSPR) and the metallic substance is termed as plasmonic nanoparticle (PNP). During the enhancement process, the LSPR generated at the surface of the PNP couples with the incident light's resonance which result to huge signal generation [63]. The magnitude of this enhancement has been determined to 
reach from $10^{3}$ to $10^{10}$ [26]. This phenomenon has led to significant effort to improve the analytical performance of SERS techniques. Today many articles [64, 65] and reviews [66, 67] have been published, all aiming at controlling the size, structures and distance of the PNP to provide reproducible fabrications of optimal conditions for highly sensitive analytical analysis.

The most common metals found to observe this phenomenon are gold and silver. Gold has been preferred over silver in the analysis of biological compounds due to its longerexcitation wavelength (above $600 \mathrm{~nm}$ ) and excellent biocompatibility [67].

Incident light

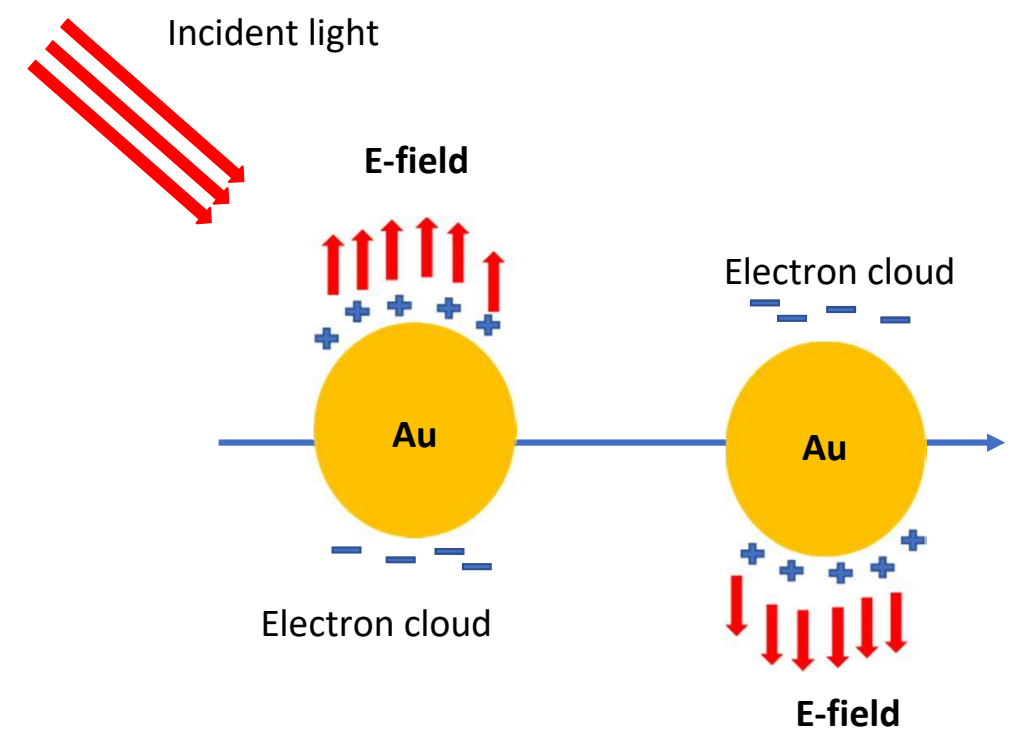

Figure 5. Electromagnetic effect of SERS. Incident light set the electron clouds into oscillation to generate large local field, two NP at a closed proximity increased in oscillation due to coupling of local electric field which enhanced signal.

\section{SERS Hot Spots}

Development of SERS technology to detect single molecules has been reported in several papers $[68,69]$ and it has been found that aggregated nanoparticles produce higher enhancement 
than a single plasmonic nanoparticle $[70,71]$. The enhancement contribution of the aggregated nanoparticle has been attributed to the interparticle distance between two PNP that are brought to closed proximity to each other. The space that is created by the two nanoparticles generates a very high electric field (as shown by Figure 5) as a result of the interaction of the electron clouds surrounding the particles and therefore produced an extremely strong SERS signal. The volume of the space that creates this strong SERS signal is referred to as a SERS-hot spot [72, 73]. These advantages have enabled various applications of SERS such as noninvasive chemical detection and identification of biological molecules such as proteins [74] and DNAs [75].

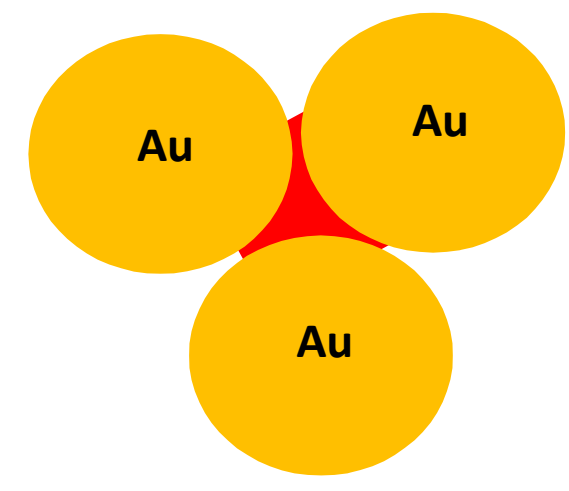

Figure 6. Illustration of SERS-hot spot (red). Generated due to decrease in interparticle distance.

\section{SERS-Based Immunoassay}

Recent developments of SERS-based immunoassays have been focused on increasing the sensitivity of the SERS substrate for various applications [76, 77]. One area that has been intriguingly successful and gaining much attention is PNP-enabled SERS immunoassay [76, 78]. The SERS active surface is fabricated by carefully arranging a gold nanoparticle array which generates the localized surface plasmon resonances and the laser exposure to the analytic biomolecules. The main focus of the SERS technology is to control the interparticle gap and 
diameter of the metal nanoparticles in order to generate SERS hot spots which increase the SERS signal when analytic biomolecules are close to the SERS-active surface.

A recent report indicates that SERS hot spots can be generated when the interparticle distance is less than $10 \mathrm{~nm}$ [79]. The detection of biomolecules using gold nanoparticle SERS strategy has been used in various applications. For example, Wang et al. reported the use of Raman label bound to an antibody-functionalized gold nanoparticle to indirectly detect rabbit IgG [35]. Also, there have been SERS immunoassay reports on the quantitation of HIV-1 [45] and staphylococcal enterotoxin [80].

Current developments in SERS-based immunoassays are focused on coupling SERS with POCT immunoassay platforms. For example, Clark et. al reported on coupling SERS with RVF for the detection of hepatitis $\mathrm{C}(\mathrm{HCV})$ biomarker using a nitrocellulose membrane as the capture substrate [51], however their technique suffers from limited sensitivity because of non-coupling of gold nanoparticles.

There have been several reports describing the plasmonic coupling in interacting AuNPs and has been found that the enhancement generated from the nanoparticle gap increased as the gap between the interacting nanoparticles decreases [81, 82]. Also, Wulstholz et al [97] reported that the enhancement is independent of the cluster size and excitation wavelength and that SERS enhancement increases with decreasing interparticle distance. Guerrini et al. also reported through the use of DNA triplexes to control the interparticle distance in nanoparticle clusters in suspension and found that the SERS enhancement correlated with nanoparticle gap distance rather than cluster or plasmon band [83]. There are several other publications which support the development of SERS as a quantitative analysis technique based on aggregation assays [84]. 
Thus, a SERS-based immunoassay that capitalizes on these nanoparticle-nanoparticle interactions would offer substantially improved sensitivity.

The SERS-based immunoassay consists of three main parts, the capture substrate, the analyte (antigen to be detected) and a functionalized gold nanoparticle. The capture substrate consists of a primary antibody immobilized on the surface of a support which selectively captures the specific antigen from the sample solution. The analyte is the antigen within the sample solution which is under investigation; they are usually macromolecules such as DNA or protein. The third component is composed of a gold nanoparticle which has a secondary antibody immobilized on its surface and is functionalized with a Raman reporter molecule in a system called the extrinsic Raman-label (ERL).

The ERL is an essential part of the assay which is normally used as a tracer molecule in the SERS-based assay to identify the presence of the analytes. The characteristic of the Raman spectrum produced is determined by the Raman reporter molecule which is used to identify the analytes. The narrow spectral width of the vibrational Raman band enables all SERS labels to be simultaneously excited with a single laser which gives advantage for SERS to identify many particles simultaneously. Examples of Raman labels used in SERS-immunoassays are 4nitrobenzenethiol (NBT) and para-aminothiophenol (p-ATP) [85].

There have been extensive on reports nanoparticle (NP) enabled SERS immunoassays for bioanalytical applications, and they have been used for diagnostic applications [86]. The first SERS-based immunoassay was developed by Ni et al. in 1999. Here, a glass slide was coated with gold film and the antibody immobilized on it, analyte is then incubated with the antibody and washed off excess reagents followed by adding the ERL and then washed again to remove nonspecific binding. The sandwiched formation is then detected using SERS. The procedure is 
simple and sensitive but has long preparation time due to slow movement of large mass of analytes (antigen) to bind with the antibody during incubation process, which can take overnight.

The ideal SERS-based immunoassay for point-of-care testing should be rapid, sensitive, easy-of-use and affordable. The speed of immunoassay plays a vital role in the diagnostic arena especially in a point-of-care testing. At the intensive care unit (ICU), POCT is used to diagnose patients with various life-threatening conditions, and the faster the diagnosis the sooner the right medical treatment response. Recent advancement in the field has been showing significant effort to speed up the SERS-based immunoassay through various ways. Our group previously developed a system using a syringe to facilitate the diffusional mass transport of analytes [87]. However, this technique also suffers from imprecision because of variable flow rate. A vertical flow system which is fast and ensures a consistent flow of solution was reported by Clarke et al, however, the technique is not sensitive due to non-coupling of nanoparticles [51].

An assay format that is highly sensitive to diagnosis trace amounts of analytes is on high demand. Recent developments towards increasing sensitivity of SERS-based assays has been focusing on improving the nanoparticle that are used as SERS-substrate [88].

\section{SERS Substrates}

SERS is a technique that involves the emission of radiation as a result of inelastic scattering of incidence laser energy that impinges on the surface of a nanometallic object which results in spectral peaks due to the vibrational modes of the molecule that are frequency shifted from the incident energy.

SERS substrate is the metallic object on which the analyte molecule is adsorbed and detected. Almost all polyatomic species exhibit Raman or SERS spectral emission. A typical 
example of SERS substrates are roughened silver, copper and gold surfaces. These SERS substrates were in the form of colloids and foils/wires, however, recent development in the SERS substrate reveal three main classifications, nanoparticle suspension, immobilized nanoparticle on solid support and fabricated nanoparticle on substrates by special treatment such as lithography synthesis. Several papers have demonstrated the use of these substrates for various applications.

Our focus is on the immobilization of metallic nanoparticles on a solid support and more emphasis will be laid on immobilization on filter paper. Aggregation of spherical particle on a solid support ensures maximum SERS enhancement [14] due to formation of SERS-hot spots. Immobilization of the nanoparticles on a solid support provides a means of this aggregation. Several immobilization strategies have been used to chemisorb nanoparticles on a solid support. Recently, immobilization of substrates on paper methods have gained attention due to the high flexibility, biocompatibility, biodegradability and low cost [50], and many papers have been reported [50, 89]. For example, Fierro-Mercado et al, reported on a highly sensitive filter paper substrate for SERS trace explosives detection [90]. In that work a thermal inkjet printer was used to print colloidal nanoparticles onto filter paper.

The SERS signal enhancement is achieved by controlling the plasmonic properties and morphology of the nanostructures that are used as SERS substrates. As such, significant efforts are underway toward an architectural modification of the plasmonic particles with new methods of fabrications to enhance SERS signal. This process is tedious and requires many steps and therefore is time consuming that limits SERS application as a fast and cheap technique. Therefore, the need to develop a rapid, sensitive and easy-to-use platform to detect biomarkers is required to advance disease diagnostic arena for the management of disease. 


\section{Paper-Based Assay (PBA)}

The use of paper-based material as SERS substrates has gained much attraction toward development of immunoassay due to the high flexibility, biocompatibility and cost effectiveness. As such, three formats have been developed including LFA, dipstick assays, and microfluidic paper based analytical devices ( $\mu$ PADs) [91].

The most common substrates that are used for the PBA are nitrocellulose membrane, filter paper, chromatography paper, and or paper/nanomaterial composites [92]. The optical properties and surface chemistry, as well as the porosity of these paper-based assays are very essential in diagnostic application. Among these papers, the most widely used is nitrocellulose membranes due to its broad distribution of pore sizes $(0.05-12 \mu \mathrm{m})$ [8] for many proteins and DNA applications. However, they have some limitation such as large accumulation of charges under low humidity [93] which prevents its ease of use. The current limitation of the PBA needs to be critically improved by signal enhancement strategies [94] as it is inappropriate for diagnostic applications. Toward this end, the need of new methods to evade nonuniformity, poor reproducibility and variable accuracy of PBA is required.

\section{Thesis Objectives}

The goal of this research is to develop a highly sensitive point-of-care system that is fast, cheap and easy to use by anyone. Here we develop a new strategy through direct addition of second gold nanoparticle layer on the plasmonic paper to create a new sensing environment that would be able to enhance the SERS signal. The procedure has the advantage of simplifying the assay procedure to less than 2 minutes and improves sensitivity of the assay by an order of magnitude. This process overcomes the limitations of LFA. 
The system consists of an antibody-gold nanoparticle conjugate functionalized with a Raman reporter molecule which is used to form a second layer on a modified antibody-AuNP plasmonic filter paper after capturing antigen from a sample that vertically flows through the plasmonic antibody-capture filter paper (Figure 7). The Raman reporter molecules provide a vibrational spectrum that indicates the presence of antigen in the sample solution. They are strategically designed to chemisorb as a thiolate adlayer on the gold nanoparticle, to provide a strong and unique spectral signature, and to covalently link a layer of the antibody to the gold nanoparticle. The vibrational spectrum resulting from the Raman reporter molecule is used to trace and quantify any captured antigen from the flow through sample.

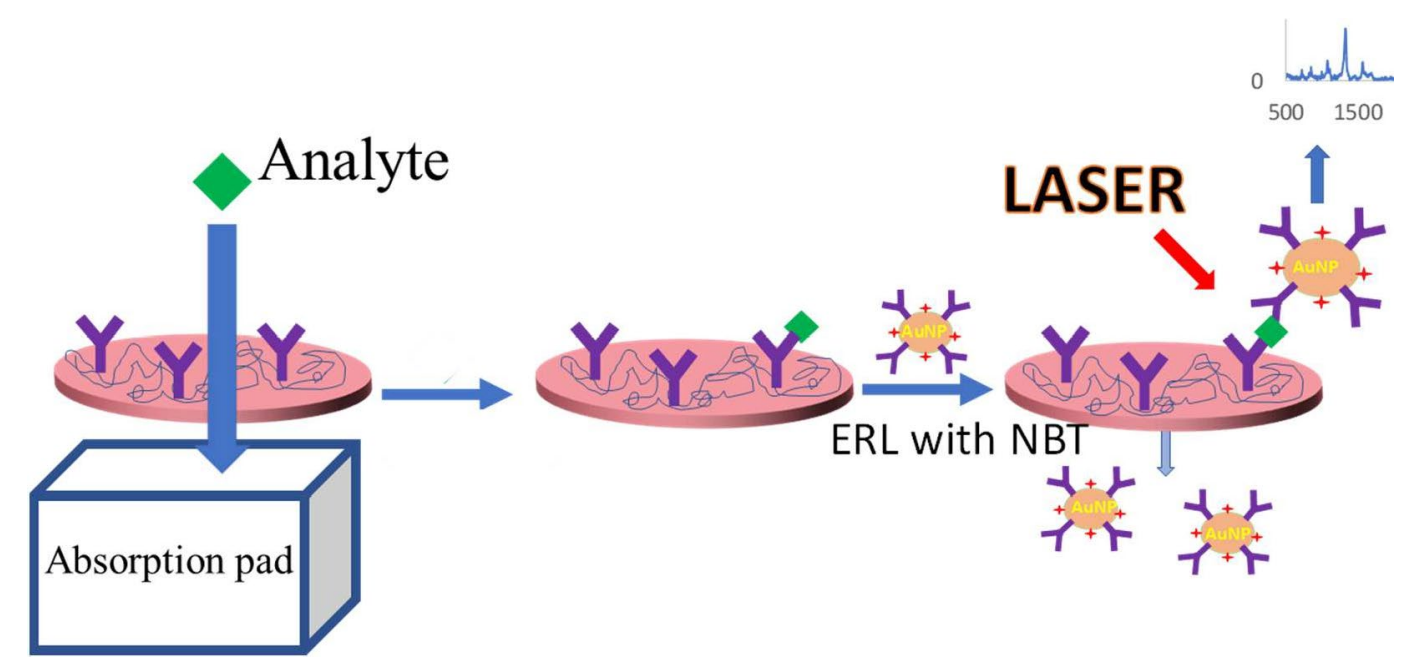

Figure 7. Overview of SERS VFA. Sample is applied and antigen is captured, ERL is applied to labelled captured antigen then analyzed with SERS. 


\section{CHAPTER II: MATERIALS AND METHODS}

All materials, reagents and various analytical procedures for this research have been explained in this chapter including various synthesis and physical measurements such as: synthesis of gold nanoparticles for fabrication of plasmonic paper, preparation of the AuNPantibody conjugates and all analytical instruments used.

\section{Materials and Reagents}

Gold nanoparticles (AuNPs; $60 \mathrm{~nm}$ ) were purchased from Ted Pella Inc. and used to synthesis extrinsic Raman labels (ERLs). Bovine serum albumin (BSA), 4-nitrobenzenethiol (4NBT), Tween 20, and mouse IgG (I5381) were purchased from Sigma-Aldrich. Goat anti-mouse IgG polyclonal antibody (ab 7063) was obtained from Abcam. Normal mouse serum was acquired from Santa Cruz Biotechnology. Phosphate buffered saline (PBS; $10 \mathrm{mM}, \mathrm{pH}$ 7.4) and sodium borate buffer $(50 \mathrm{mM}, \mathrm{pH} 8.5)$ were purchased from Thermo Scientific. Whatman filter paper grades 4 and 40 were used to prepare SERS substrate. A vertical flow device (Zoom Blot Plate) and high capacity absorbing pads were purchased from Vitrozm.

\section{Synthesis of Gold Nanoparticles (AuNPs)}

AuNPs were synthesized and subsequently used to prepare plasmonic paper. Briefly, AuNPs with $\square 60 \mathrm{~nm}$ diameters were synthesized by the modified thermal reduction method $[95,96]$. An aliquot $(2.0 \mathrm{~mL})$ of gold solution $\left(1 \mathrm{wt} \% \mathrm{HAuCl}_{4} \cdot 3 \mathrm{H}_{2} \mathrm{O}\right)$ was diluted to $100 \mathrm{~mL}$ with water in a 250 $\mathrm{mL}$ Erlenmeyer flask containing a magnetic stirring bar. After vigorously stirring the solution for 15 min, the flask was heated to boiling. Trisodium citrate $(1 \mathrm{wt} \% ; 1.5 \mathrm{~mL})$ was quickly added to the boiling solution, which led to the formation of spherical AuNPs. After completing the reaction, the 
final solution was adjusted to $80 \mathrm{~mL}$ in total volume (exhibiting an extinction maximum of $\square 2.4$ ) and stored at room temperature without further purification prior to use.

\section{Preparation of Plasmonic Paper Capture Substrate}

Whatman grade 4 and 40 filter paper with 8 and $25 \mu \mathrm{m}$ pores sizes, respectively, were selected to prepare the plasmonic paper capture substrate. Prepared AuNPs were embedded in the two filter papers using a previously developed dipping method [97-100]. The papers were first dried at $50{ }^{\circ} \mathrm{C}$ in an oven overnight and then immersed in $10 \mathrm{~mL}$ of the synthesized AuNP suspension in a plastic petri dishes $(60 \mathrm{~mm} \times 15 \mathrm{~mm})$. After soaking for $24 \mathrm{~h}$, the filter papers were removed from the AuNP suspension and completely dried in the oven $\left(\sim 40^{\circ} \mathrm{C}\right)$ for $1 \mathrm{hr}$. The resulting plasmonic paper was then cut into circles with a $3 \mathrm{~mm}$ diameter for use in the SERS immunoassay. Unless noted otherwise, $2 \mu \mathrm{L}$ of $1 \mathrm{mg} / \mathrm{mL}$ goat anti-mouse antibody was applied to the $3 \mathrm{~mm}$ plasmonic paper substrate and dried in a desiccator for $1.5 \mathrm{~h}$ before use [101].

\section{Preparation of Extrinsic Raman Labels (ERLs)}

Antibody and a Raman reporter molecule were co-adsorbed onto AuNPs to prepare extrinsic Raman labels (ERLs), following a previously reported procedure [102, 103]. A $1.0 \mathrm{~mL}$ aliquot of $60 \mathrm{~nm}$ AuNPs (Ted Pella, Inc.) was added to a microcentrifuge tube and $40 \mu \mathrm{L}$ of 50 $\mathrm{mM}$ borate buffer ( $\mathrm{pH} 8.5)$ was introduced to adjust the $\mathrm{pH}$. Goat anti-mouse IgG antibody (30 $\mu \mathrm{g})$ and $10 \mu \mathrm{L}$ of $1 \mathrm{mM}$ 4-NBT were added and incubated for 1 hour to allow the antibody and 4-NBT to adsorb onto the AuNPs, forming the ERLs. The ERL suspension was centrifuged at $5000 \mathrm{~g}$ for $5 \mathrm{~min}$ and the supernatant above the pelleted ERLs was discarded to remove excess

antibody and 4-NBT that was not adsorbed to the AuNPs. The ERLs were resuspended in $2 \mathrm{mM}$ 
borate buffer and the centrifugation/resuspension cycle was repeated two additional times. To the purified ERLs, $10 \mu \mathrm{L}$ of $10 \%$ BSA was added to further passivate any remaining surface on the AuNPs and $10 \mu \mathrm{L}$ of $10 \% \mathrm{NaCl}$ was added to mimic physiological ionic strength and prevent protein unfolding.

\section{Vertical Flow Immunoassay Protocol}

Calibration standards of the antigen were prepared by diluting a $1 \mathrm{mg} / \mathrm{mL}$ stock solution of mouse IgG to final concentrations of $0.1,0.5,1,10,50,100,200,300$ and $400 \mathrm{ng} / \mathrm{mL}$. Two sets of calibration standards were prepared, one using PBS as the diluent and another using rabbit serum as the diluent. PBS or rabbit serum served as the negative control to assess nonspecific binding and specificity. The capture substrate, comprised of the plasmonic filter paper with preadsorbed goat anti-mouse IgG antibody, was then loaded on the adsorbing pad and inserted into a vertical flow device. Once assembled, 1\% BSA $(100 \mu \mathrm{L})$ was added to the sample well, and rapidly passed through the capture substrate into the absorbing pad to block the capture substrate and sample well surface in an effort to prevent nonspecific binding. The sample solution, e.g., calibration standard or normal mouse serum, was then added to the sample well and antigen molecules were captured on the plasmonic paper by the immobilized antibody as the solution freely passed through the capture substrate. Next, ERLs were added to the sample well and passed through the capture substrate to label any captured antigen, with excess ERLs drawn into the absorbing pad. Excess ERLs were removed from the surface of the plasmonic paper by passing a PBS rinse solution containing 5\% Tween 20 through the paper. The plasmonic paper was removed and allowed to dry in a desiccator before SERS analysis. 


\section{Characterization Methods}

The following sections provide details of characterization techniques and the detail of procedures that were used to evaluate the performance of the assays, these include: Scanning electron microscope (SEM), Surface enhanced Raman spectroscopy (SERS), Dynamic light scattering (DLS) and Ultraviolet-visible spectroscopy.

\section{Scanning Electron Microscope (SEM)}

The plasmonic papers were examined using FEI-Quanta $450 \mathrm{SEM}$ operating at $20 \mathrm{kV}$ to produced visual images of the papers. Prior to this analysis, the papers were coated with a thin gold film using a Denton vacuum sputter coater (DESK II), to avoid common charging problems. The images were collected to measure the general size distribution of plasmonic particles and to visualize overall packing patterns of the NPs on the surface of the filter paper.

\section{Surface Enhanced Raman Spectroscopy (SERS).}

To evaluate SERS response of the vertical flow assay, SERS spectra were collected using an Enwave Optronics, Inc. ProRaman-L-785B instrument configured with a $785 \mathrm{~nm}$ excitation source set to $10 \mathrm{~mW}$ at the sample surface and a high-sensitivity CCD thermoelectrically cooled to $-60{ }^{\circ} \mathrm{C}$. The sample was placed on an $\mathrm{x}-\mathrm{y}-\mathrm{z}$ sample stage and the laser was focused on the substrate surface by maximizing the SERS intensity. After focusing the sample, the stage was automated to move linearly along the x-direction during the $10 \mathrm{sec}$ spectral acquisition as a means of increasing the sampling area on the capture substrate, effectively averaging signal from different locations on the substrate [104]. Five spectra were collected from each sample substrate 
and baseline corrected using the auto-baselin-2 algorithm built into the Enwave application software (ProRaman Reader V8.2.8).

\section{Dynamic Light Scattering (DLS)}

The adsorption of the antibodies on the AuNPs of the ERL were confirmed by measuring the mean hydrodynamic diameter and polydispersity of the AuNPs before and after the preparation of the ERLs with a Malvern Zetasizer Nano ZSP. Unconjugated AuNP and synthesized ERLS were diluted 2-fold with nano pure water and placed in a micro-volume disposable Eppendorf cuvette for DLS analysis. The sample was equilibrated for $60 \mathrm{sec}$ prior to analysis and each size measurement was determined from 10 runs, 10 sec each.

\section{UV-Visible Spectrometer}

The synthesized gold nanoparticles used to prepare the plasmonic paper were investigated to assess the number of AuNPs loaded on the filter paper by measuring the extinction of the AuNP suspension before and after adsorption onto the filter paper. The UVvisible instrument used for this analysis was Agilent 8453 spectrophotometer (Agilent Technologies, Santa Clara, CA). The instrument has a photodiode detector with a spectral range of $190-1100 \mathrm{~nm}$. Prior to sample analysis the instrument was blanked with $2 \mathrm{mM}$ borate buffer (pH 8.5). The prepared plasmonic paper was also characterized using a surface UV-Vis-IR spectrophotometer $(300-1700 \mathrm{~nm})$ equipped with a reflectance probe (StellarNet). 


\section{CHAPTER III: RESULTS AND DISCUSSION}

\section{Assay Design and Sensing Principle}

A general overview of the SERS-based vertical flow immunoassay is illustrated in Figure 8. Central to this platform is AuNP-loaded plasmonic paper, which serves as a capture substrate for the detection of antigens. Antibody is pre-immobilized onto the plasmonic paper and antigen is extracted and concentrated on the substrate as the sample solution passes through to an absorbing pad via capillary action. In a second step, ERLs are spontaneously and vertically passed through the plasmonic paper to specifically label captured antigen and facilitate SERSbased detection. The micron-sized pores in the filter paper allow any excess nanometer-sized ERLs to rapidly and freely pass through the plasmonic paper to waste collected in the absorbent pad. The plasmonic paper provides two key features. First, antibody irreversibly adsorbs onto AuNPs embedded in the filter paper [105-108], to form a robust capture substrate that resists desorption and loss of function while solutions flow through the paper. Second, the AuNP embedded in the paper will form a sandwich-like structure with ERLs bound to captured antigen. This architecture greatly supports plasmonic coupling between the AuNPs to generate a large localized electric field between the sandwiched nanoparticles and significantly enhance the SERS signal relative to the isolated ERL in the absence of plasmonic coupling [98, 109-112]. In addition to the uniquely designed plasmonic paper to maximize analytical signal, the vertical flow format overcomes diffusional mass transport limitations of traditional immunoassays to substantially reduce assay time and does not suffer from the hook effect, as is the case for lateral flow assays, to improve quantitative capabilities [45].

A model antibody-antigen system was employed to establish proof-of-principle for the SERS-based LFA and illustrate the role of the plasmonic capture substrate. The plasmonic 
capture substrate and ERLs were both synthesized with goat anti-mouse IgG polyclonal antibody and mouse IgG was employed as the antigen. The following sections detail the synthesis of the plasmonic paper, the role of the plasmonic paper to facilitate large SERS enhancements, and the assay parameters that impact analytical performance. After optimization, we defined the analytical figures of merit and applied the protocol to accurately quantify IgG in normal mouse serum.
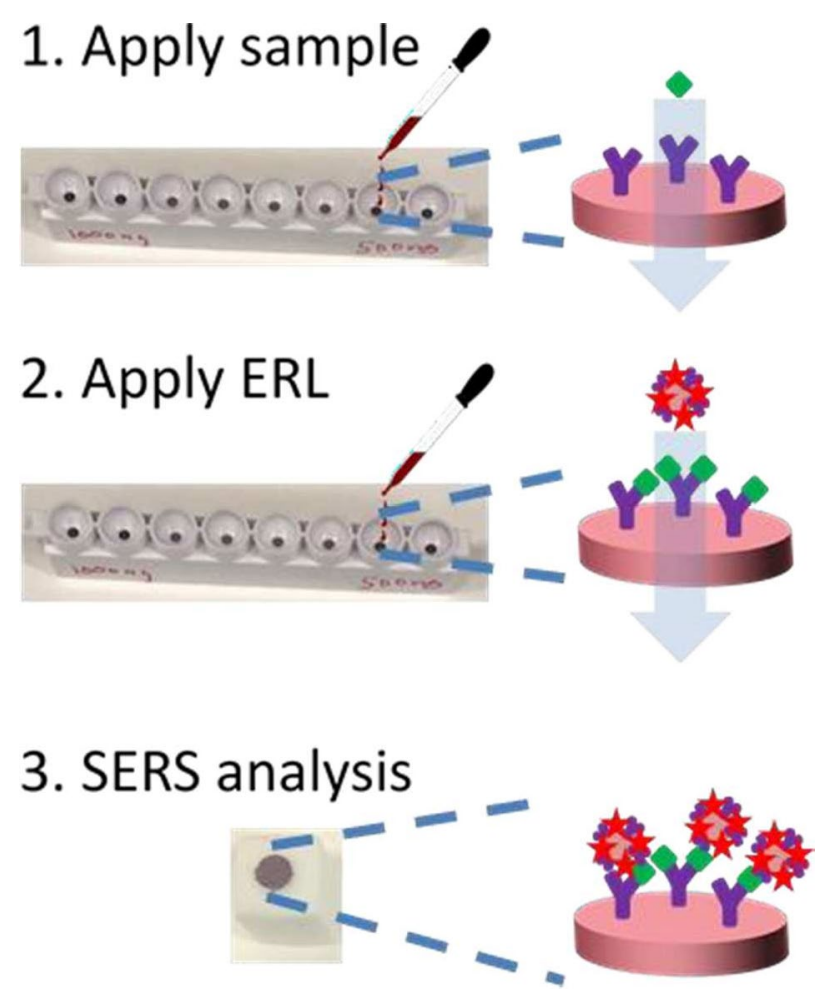

Figure 8. Illustration of the SERS-vertical flow immunoassay protocol. In the first step, the sample solution is vertically passed through the paper for antibody to extract the analyte. In the second step, ERLs are passed through paper to label the captured antigen. 


\section{Fabrication and Characterization of Plasmonic Paper}

Filter paper loaded with spherical AuNPs serve as the foundation of the capture substrate in the SERS-based vertical flow assay illustrated in Figure 8. Filter paper was selected because of its numerous advantages such as affordability, accessibility, biodegradability, disposability, modification, and variety of unique pore sizes. In this work, we selected Grade 4 and 40 Whatman filter paper with nominal pore sizes of $25 \mu \mathrm{m}$ and $8 \mu \mathrm{m}$, respectively. Figure 9 shows digital photographs of the two filter papers before and after loading with AuNPs, prepared by dip coating [97-100]. The change in color of the filter paper from white to purple indicates the adsorption of the AuNPs on the paper, and the consistent color across the full paper suggests uniform distribution on a macroscopic scale. SEM images confirm adsorption of the AuNPs onto the fibers of the filter paper with a mean diameter of $57.9 \pm 12.5 \mathrm{~nm}$. The images further reveal small aggregates consisting of a few AuNPs are formed on the filter paper during the dip coating process. Moreover, the SEM images show a greater loading density of AuNPs on the grade 4 filter paper than the grade 40 filter paper. The AuNP adsorption efficiency was quantified by the changes of AuNP surface plasmon resonance (SPR) using UV-visible spectrophotometry. The AuNP suspension exhibited an extinction of 2.4 at $540 \mathrm{~nm}$ before filter paper was dipped intothe suspension. After immersing the filter paper in the AuNP suspension for $24 \mathrm{~h}$ and subsequent removal, the remaining suspension displayed an extinction of 0.7 and 1.3 for the grade 4 and 40 filter papers, respectively (Figure 9). These decreases in SPR bands represent the loading of 2.8 $\times 10^{11}$ AuNPs and $1.8 \times 10^{11}$ AuNPs on the grade 4 and 40 filter papers, respectively. 

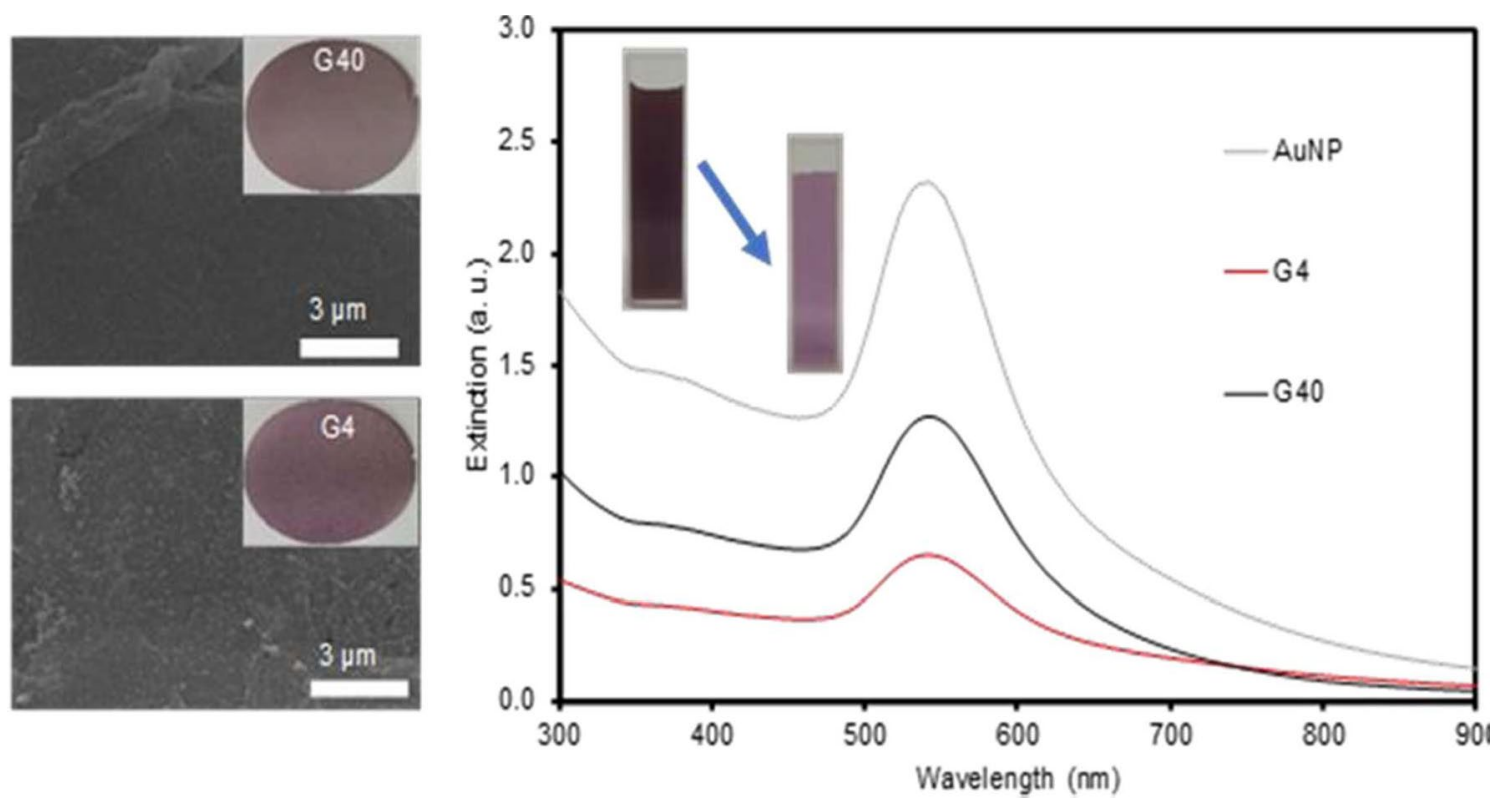

Figure 9. SEM images of plasmonic filter papers with their UV analysis of AuNPs solution of grade number 4 and 40 with their corresponding UV analysis of before and after loading on the papers.

To support the detectably, different amounts of plasmonic AuNP loading onto these two papers, the surface UV-Vis-IR absorption patterns were obtained (Figure 10). Although obtaining surface absorption patterns by a reflectance probe is somewhat difficult due to the light absorbing characteristics of materials, the clearly different SPR patterns (i.e., broader SPR band at $545 \mathrm{~nm}$ and increased background peak over $700 \mathrm{~nm}$ ) explained that the grade 4 filter paper possessed a relatively higher number of locally aggregated AuNPs than the grade 40 filter paper. The presence of slightly more and dense packing of AuNPs could induce a higher probability of effective plasmonic coupling that can favorably influence the degree of SERS enhancements. 


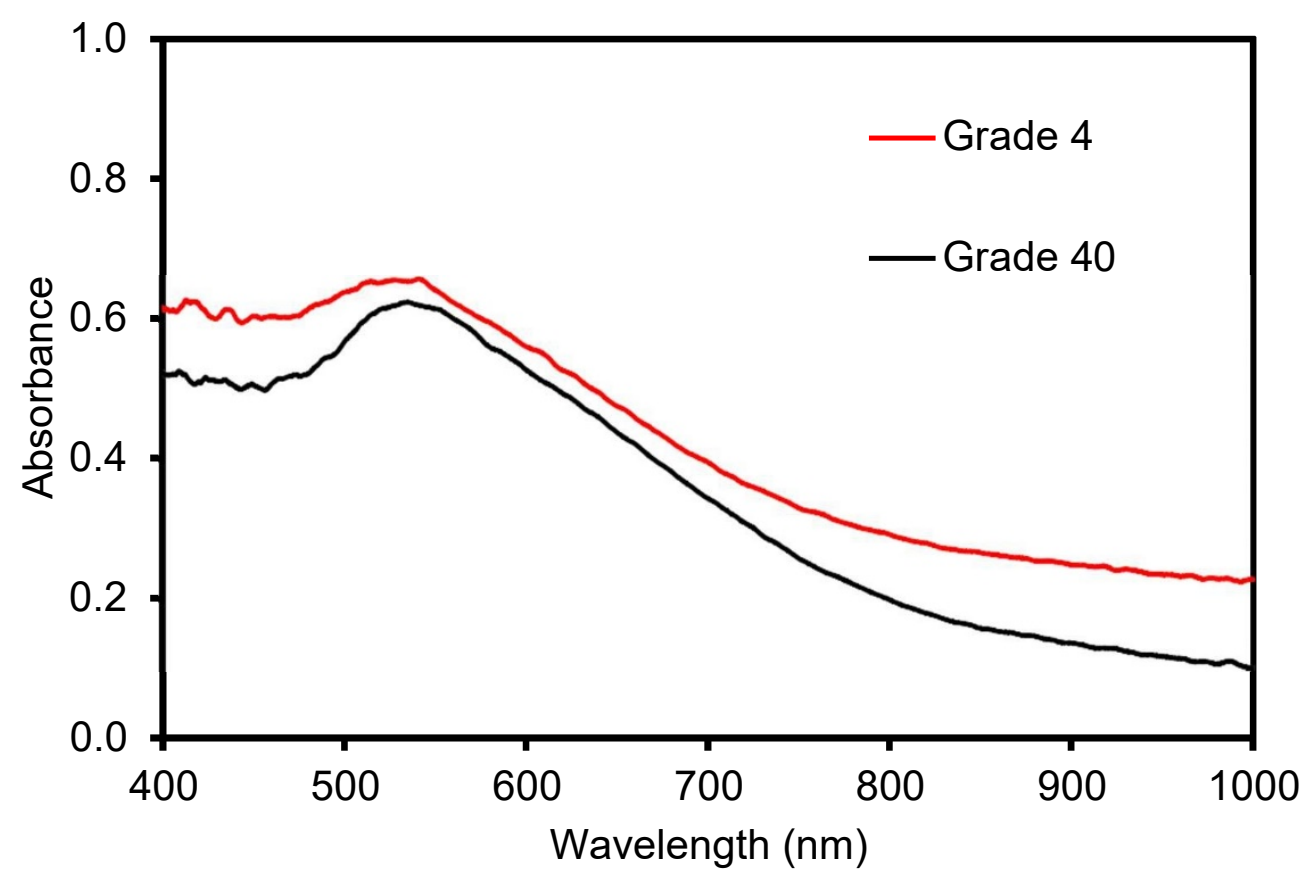

Figure 10. Surface UV-Vis-IR spectra of AuNPs loaded onto plasmonic papers of grade number 4 and 40 filter papers.

\section{Role of Plasmonic Paper}

SERS-based vertical and lateral flow immunoassays have been previously developed using paper and nitrocellulose membranes as capture substrates. The goal of this work is to capitalize on the benefit afforded by a plasmonic capture substrate to create sandwich-like structures and achieve greater signal enhancements. To establish the value of the plasmonic capture substrate, the vertical flow assay was performed using unmodified filter paper and plasmonic filter paper. Antibody was deposited on unmodified filter paper and AuNP-embedded filter paper to form the capture substrate $[113,114]$. After blocking the paper with BSA, $100 \mu \mathrm{L}$ of the positive control sample ( $100 \mathrm{ng} / \mathrm{mL}$ of mouse $\operatorname{IgG})$ was passed through the capture substrate. The sample solution quickly passed through the unmodified filter paper and grade 4 
plasmonic paper in less than $10 \mathrm{sec}$; however, the $100 \mu \mathrm{L}$ sample required more than 2 min to flow through the grade 40 plasmonic paper. After allowing the sample to completely pass through the capture substrate to the absorbent pad, $100 \mu \mathrm{L}$ of ERLs were precisely applied to the sample and allowed to pass through the capture substrate to label the antigen extracted by the capture substrate. Again, the ERL flow rate was significantly faster through the grade 4 plasmonic paper than the grade 40 plasmonic paper. After rinsing the paper with PBS $(100 \mu \mathrm{L})$, SERS spectra were collected to quantify the bound antigen. Representative SERS spectra are presented for each of the capture substrates in Figure 11. As is evident, the SERS signal is characteristic of the Raman reporter molecule, 4-NBT, used to synthesize the ERLs, and include prominent bands at $1338 \mathrm{~cm}^{-1}$ (symmetric $\mathrm{NO}_{2}$ stretch), $1559 \mathrm{~cm}^{-1}(\mathrm{C}=\mathrm{C}), 1153 \mathrm{~cm}^{-1}(\mathrm{C}-\mathrm{O})$, and $850 \mathrm{~cm}^{-1}(\mathrm{C}-\mathrm{H})[115,116]$. Importantly, the signal is markedly increased for the plasmonic paper capture substrates relative to the capture substrate prepared with unmodified filter paper (Figure 11). Plasmonic paper resulted in $\sim 4$-fold and $\sim 10$-fold increase in signal for the capture substrate prepared with grade 40 and grade 4 filter paper, respectively, relative to the unmodified paper. Negative control samples were also analyzed to confirm that the signals obtained for the mouse IgG sample was due to specific antibody-antigen interactions rather than non-specific binding of the ERLs (e.g., physical adsorption) in the filter paper used as the foundation of the capture substrate. To this end, PBS was passed through the capture substrates in the first step. Subsequently, ERLs were passed through the capture substrates and analyzed via SERS (Figure 11). The signal measured for the PBS negative control was substantially lower than that observed for the analysis of the positive control sample for each of the capture substrates. These results confirm the specificity of antibody-antigen interaction yet reveal some non-specific binding of the ERLs. 
Collectively, these results clearly demonstrate greater SERS signals are obtained for the plasmonic capture substrates than the unmodified capture substrate. Interestingly, the plasmonic paper fabricated with grade 4 filter paper provided greater signal for the analysis of the positive control and lower signal for the analysis of the negative control sample than the grade 40 filter paper. As indicated earlier, the SERS enhancement is as a result of interparticle coupling that generates strong localized electromagnetic fields to maximize SERS signal from the substrate. Our results suggest that SERS enhancement provided by the plasmonic capture substrate corresponds to the number of plasmonic nanoparticles that are present on the surface of the paper. Moreover, the paper with the bigger pore size, e.g., grade 4, resulted in less non-specific binding of ERLs to generate less false-positive signal. Owing to this dual benefit, Whatman filter paper grade number 4 was selected to prepare plasmonic capture substrates and optimize the assay parameters.

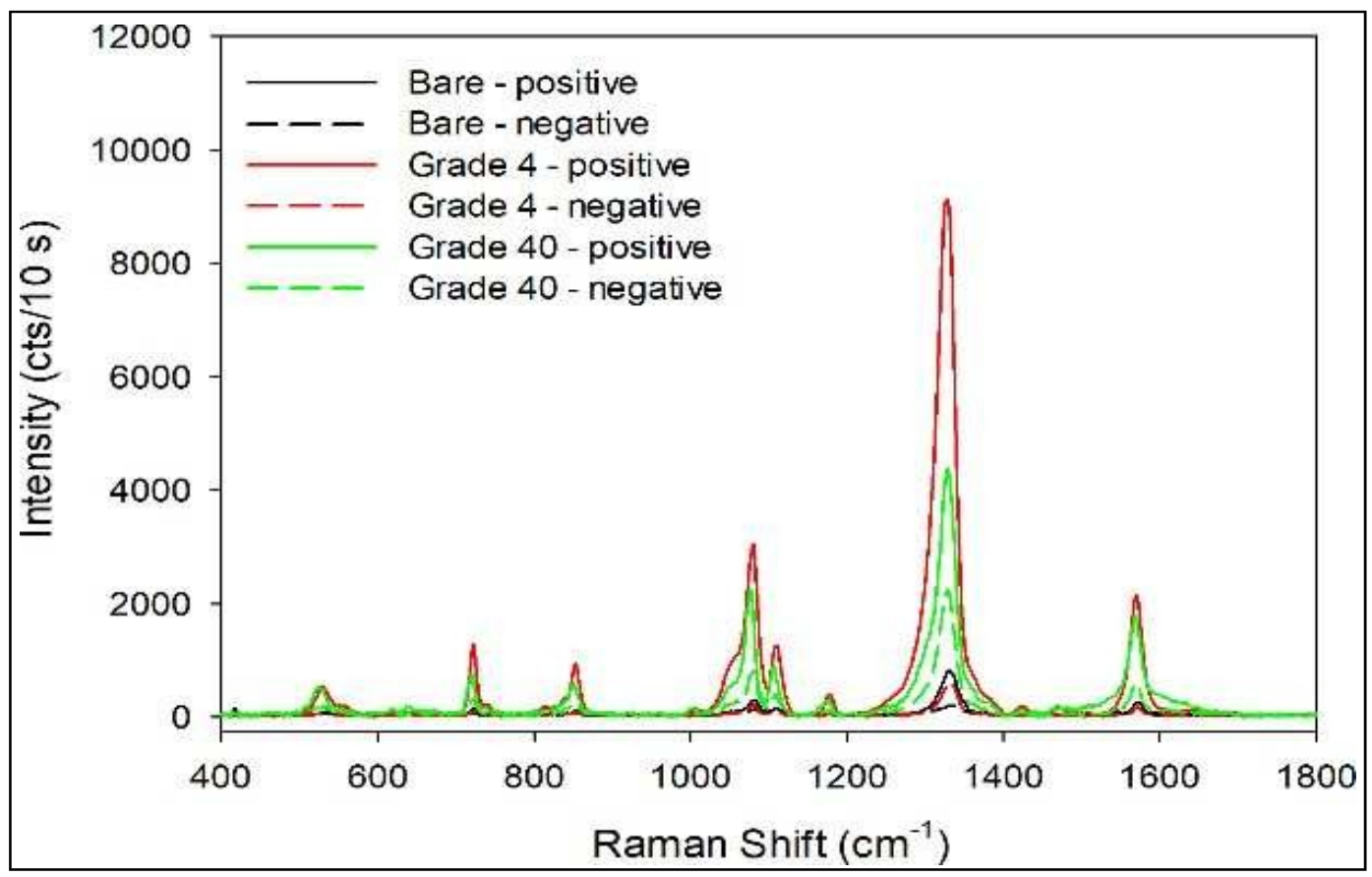

Figure 11. SERS spectra collected using bare filter paper, plasmonic filter papers of grade 4 filter paper, and grade 40 plasmonic filter paper as the capture substrate in a SERS-RVF 
immunoassay. The positive control samples represent the analysis of $100 \mathrm{ng} / \mathrm{mL}$ mouse IgG and the negative control samples represent the analysis of PBS.

\section{Optimization of Assay Protocol}

The following section explains the studies of the various assay parameters that enabled achievement of the optimized conditions which focus on varying the amount of capture antibodies, volume of ERLs and rinsing volume of PBS.

\section{Amount of Capture Antibody}

The ability to capture and concentrate antigen on the surface of the plasmonic paper as it passes through correlates with the number of capture antibodies available on the substrate. Therefore, the amount of the antibody deposited on the surface of the paper is an important parameter that can contribute to the analytical performance of the vertical flow immunoassay. Ideally, sufficient antibody is added to the plasmonic paper to saturate all available immobilization sites. However, addition of a large excess of antibody must be avoided to minimize the cost of the assay. To this end, goat anti-mouse IgG antibody (1 to $4 \mu \mathrm{g}$ ) was deposited on plasmonic paper (grade 4) to prepare capture substrates with variable amounts of capture antibody. A positive control sample (100 ng/mL antigen) and negative control sample (PBS) were analyzed with each capture substrate, using $100 \mu \mathrm{L}$ of ERLs and $100 \mu \mathrm{L}$ of PBS for subsequent labeling and rinsing, respectively. The SERS signal as a function of the amount of capture antibody is plotted for the positive and negative samples in Figure 12a. The positive control signal increased as the amount of deposited capture antibody increased from 1 to $2 \mu \mathrm{g}$; however, no further increase was observed for the positive control sample with the deposition of 
more than $2 \mu \mathrm{g}$ of capture antibody. The signal for the negative control sample was independent of the mass of capture antibody immobilized on the capture substrate. Based on the results of this experiment, $2 \mu \mathrm{g}$ of capture antibody was selected for cost effectiveness, since this mass provided enough antibodies to effectively maximize the capture of antigen in a $100 \mathrm{ng} / \mathrm{mL}$ sample and produce a maximum SERS signal.

To understand the activity of these capture antibodies, a paper to paper variability analysis was conducted which are shown in Figures $12 \mathrm{~b}$ and 12c. Each bar represents average intensity of five SERS spectra collected from five different location on the paper with error bars. The results of the two papers show the same trend with statistically equivalent SERS intensities.

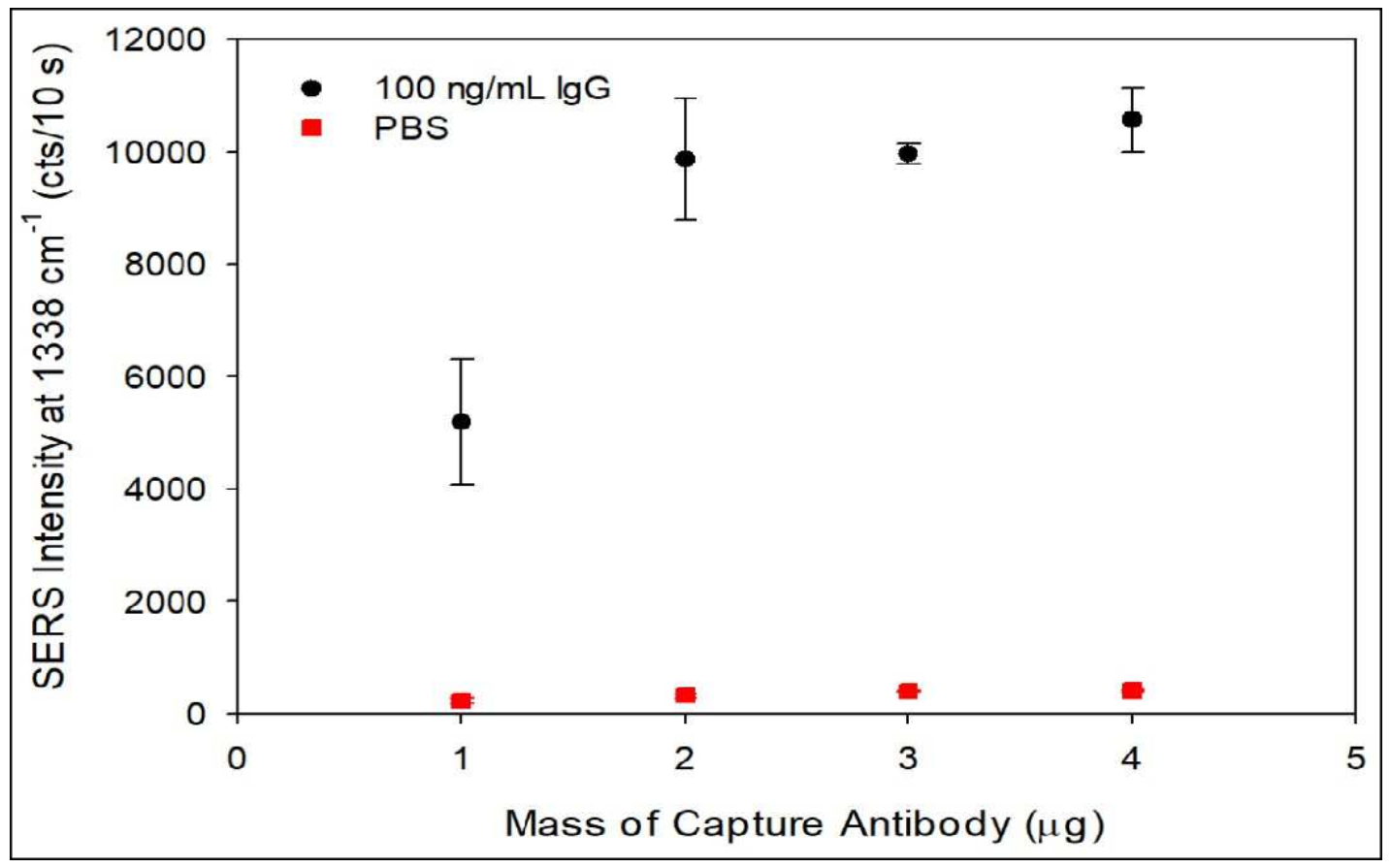



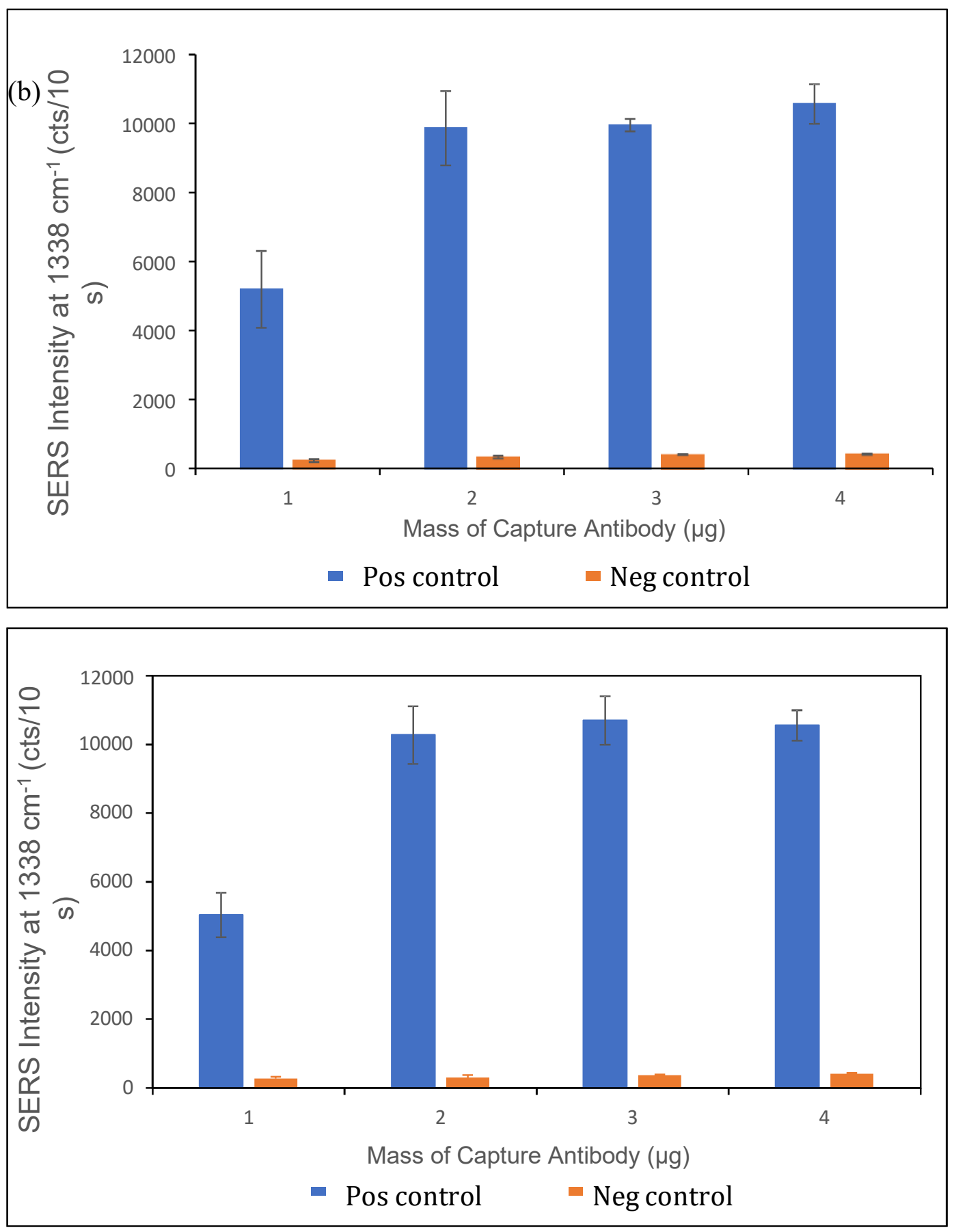

Figure 12. Effect of the amount of capture antibody on plasmonic filter paper grade number 4. obtained by varying the mass of the capture antibody to capture $100 \mathrm{ng} / \mathrm{mL}$ mouse $\mathrm{IgG}$, with subsequent labelling and rinsing using $100 \mu \mathrm{L}$ of ERLs and $100 \mu \mathrm{L}$ of PBS. (a) positive and negative control samples (b) plasmonic paper 1 (c) plasmonic paper 2. 


\section{Volume of Extrinsic Raman Labels (ERLs)}

The effect of ERL volume on SERS intensity was investigated to maximize the analytical signal by ensuring all the captured antigen was labelled. Plasmonic paper was loaded with $2 \mu \mathrm{g}$ of capture antibody to prepare the capture substrate. A positive control sample $(100 \mathrm{ng} / \mathrm{mL}$ antigen) or a negative control sample (PBS) was passed through the capture substrate followed by varying volumes of ERLs $(25,50,100$, and $200 \mu \mathrm{L})$ to label the capture antigens. After rinsing with $100 \mu \mathrm{L}$ of buffer SERS spectra were collected, and the average intensities are plotted as a function of ERL volume in Figure 13a. The SERS signal increases as the ERL volume increases from 25 to $100 \mu \mathrm{L}$, suggesting that $<100 \mu \mathrm{L}$ of ERLs is insufficient to label all of the captured antigen for a sample concentration of $100 \mathrm{ng} / \mathrm{mL}$. While there was a slight increase in the mean signal when the ERL volume increased from 100 to $200 \mu \mathrm{L}$ the difference was not statistically different at the $95 \%$ confidence level; thus, indicating that $100 \mathrm{~mL}$ of ERLs was sufficient to label all antigens captured on the substrate for the $100 \mathrm{ng} / \mathrm{mL}$ sample. It is worth noting that analysis of samples with a concentration greater than $100 \mathrm{ng} / \mathrm{mL}$ may result in more captured antigens and therefore require a greater ERL volume to fully label all antigen molecules. Importantly, the signal of the negative control samples did not statistically differ among any of the ERL volumes tested. Therefore, based on the parameters used in this optimization study, $200 \mu \mathrm{L}$ of ERLs were considered optimized and used for subsequent assays.

To understand the activity of the ERL, a paper to paper variability analysis were conducted which are shown in Figure 13b and 13c. Each bar represents average intensity of five SERS spectra collected from five different location on the paper with error bars. The results of the two papers shows the same trend with statistically equivalent SERS intensities. 
(a)

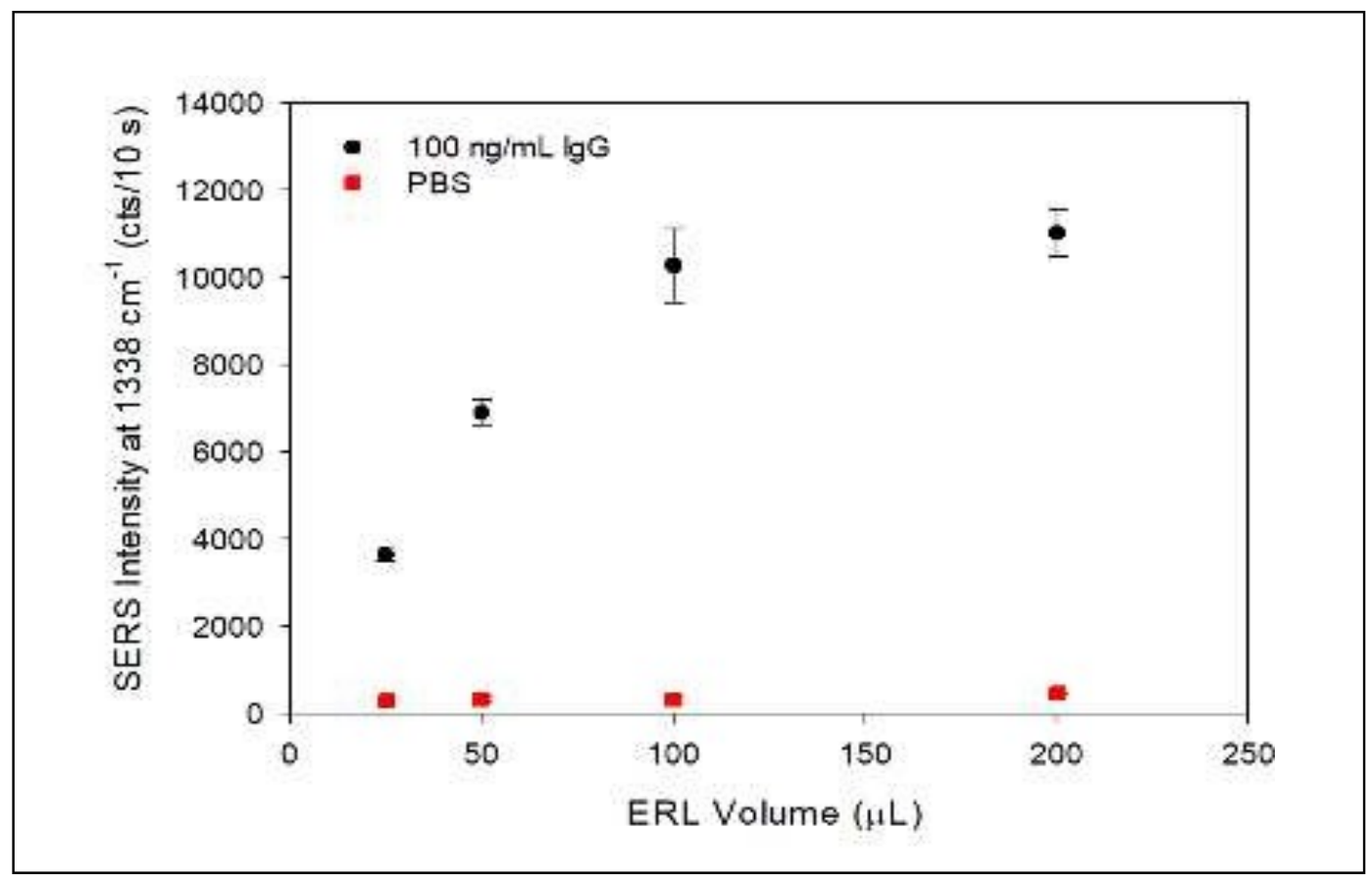

(b)

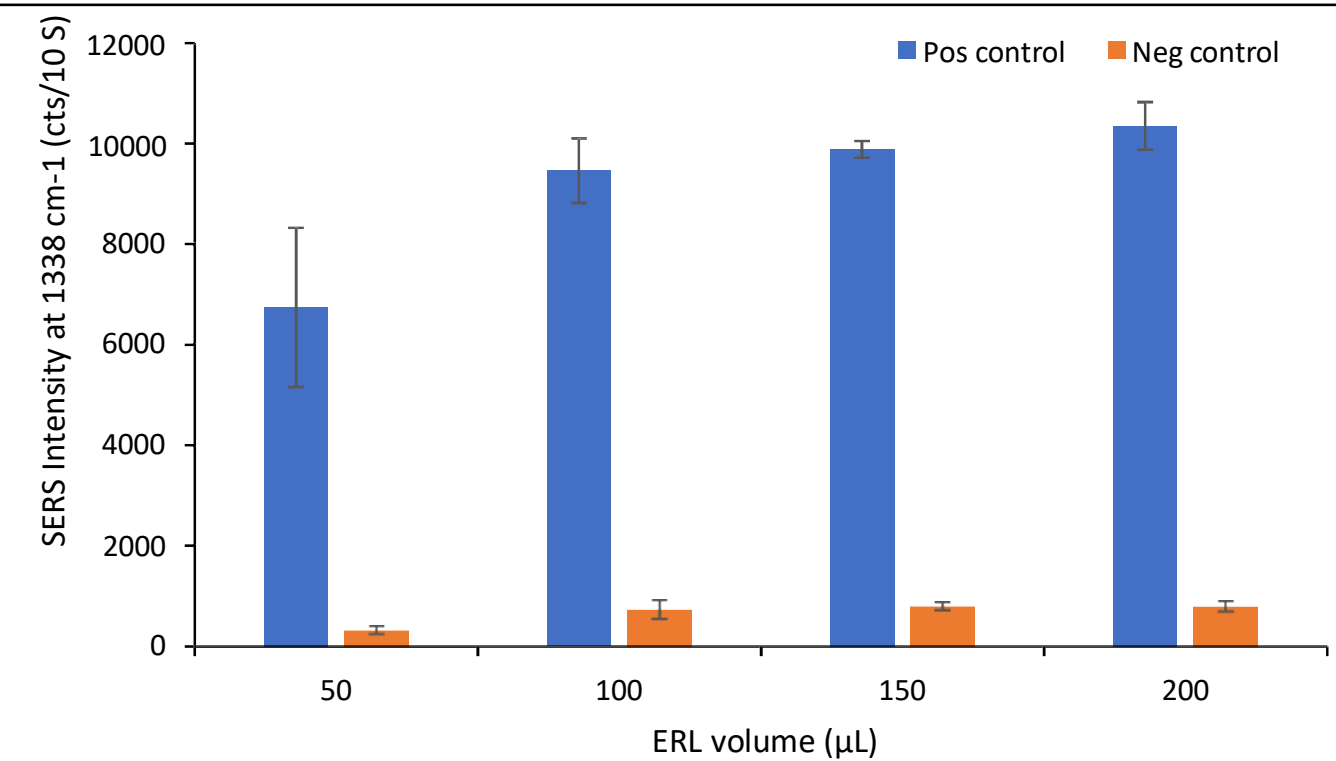


(c)

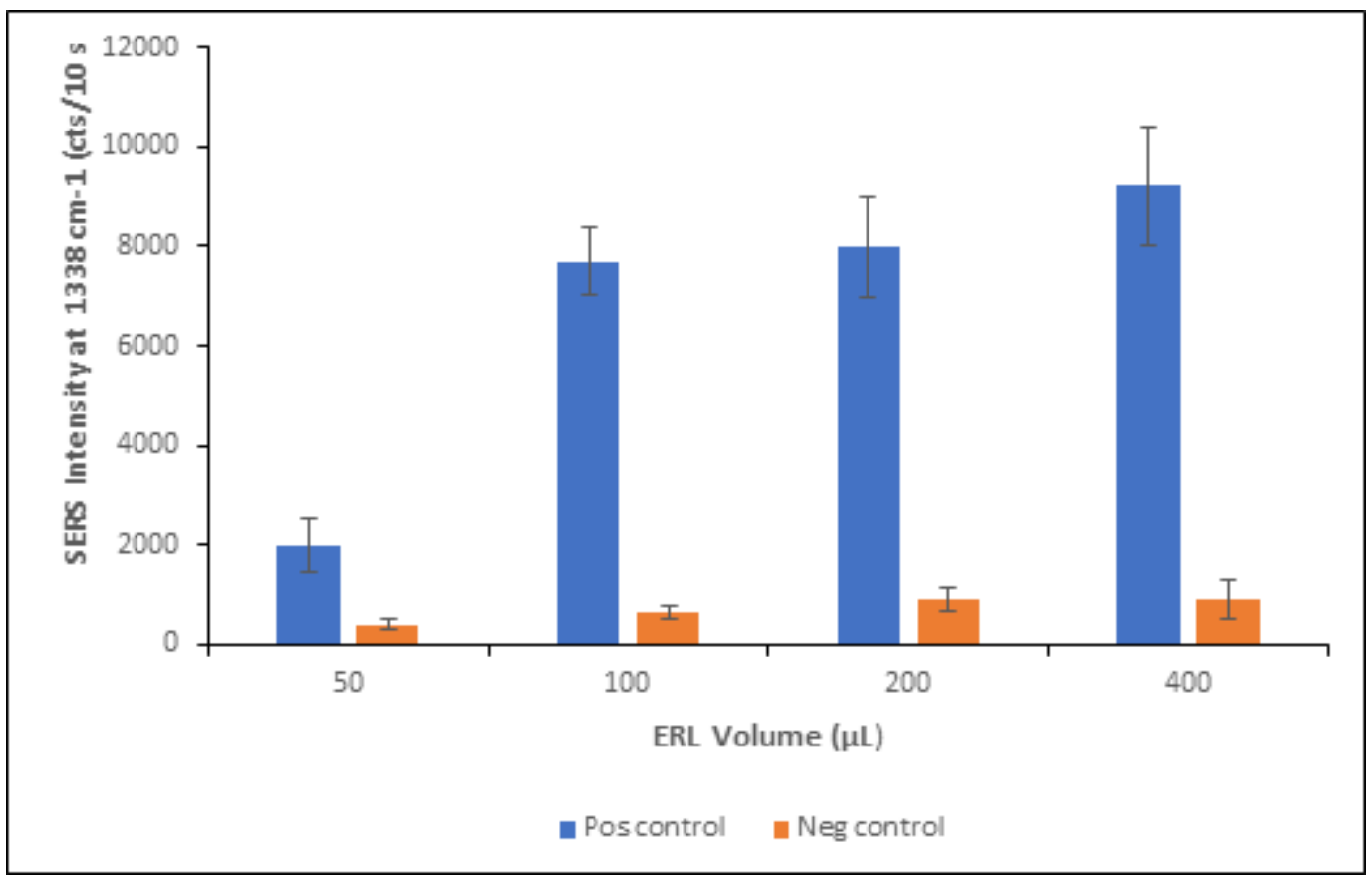

Figure 13. Effect of ERL volume on SERS intensity of plasmonic papers grade number 4 capture substrate. Obtained using $2 \mu \mathrm{L}$ of $1 \mathrm{mg} / \mathrm{ml}$ anti-goat mouse $\mathrm{IgG}, 100 \mathrm{ng} / \mathrm{ml}$ of mouse IgG and $50 \mu \mathrm{L}$ of PBS rinsing volume with variable ERL volumes. (a) positive and negative control samples (b) Paper 1 (c) Paper 2.

\section{Volume of Rinsing Buffer}

We investigated the effect of rinsing volume in an effort to minimize non-specific binding. In this study, positive (100 ng/mL antigen) and negative (PBS) were analyzed by the vertical flow assay using the optimized mass of capture antibody $(2 \mu \mathrm{g})$ and volume of ERLs (200 $\mu \mathrm{L})$. Following the labeling step, different volumes of the rinsing buffer were applied to the sample well and passed through the capture substrate to remove non-specifically bound ERLs. Figure 14a plots the SERS intensities for the negative samples as a function of rinse buffer volume. The signal for the negative control sample is a measure of the non-specific binding, and 
the data show that the negative control signal decreases as the volume of rinse buffer increases from 50 to $200 \mu \mathrm{L}$. That data clearly shows that excess unbound ERLs remain in the capture substrate filter paper when the rinse volume was less than $200 \mu \mathrm{L}$. An increase to $400 \mu \mathrm{L}$ of PBS rinsing buffer did not lead to a further reduction in the background signal. Figure 14a (inset) plots the signal collected for the positive control samples as a function of PBS rinsing volume. The data show a decrease in the signal for the positive control sample as the rinse volume increases from 50 to $100 \mu \mathrm{L}$, which is likely due to the removal of excess unbound ERLs. The positive control signal is independent of rinse volumes between 100 and $400 \mu \mathrm{L}$, which suggests that these volumes do not disrupt the specific antibody-antigen interactions. Based on the assessment of these results, we selected $300 \mu \mathrm{L}$ of rinsing volume for subsequent optimized assays. This volume of rinsing buffer ensures a thorough removal of excess, non-specifically bound ERLs and maintains the maximum analytical signal, while balancing the assay time. Larger volumes require more time for the buffer to pass through the capture substrate, and it is noteworthy that the flow rate decreases as the absorbent pad becomes saturated with liquid.

To understand the activity of the rinsing buffer, a second sample was analyzed using different prepared plasmonic paper with same rinsing volume variation and the same conditions and the results are plotted in Figure 14b. Each bar represents the average intensity of five SERS spectra collected from five different locations on the paper with error bars. 
(a)

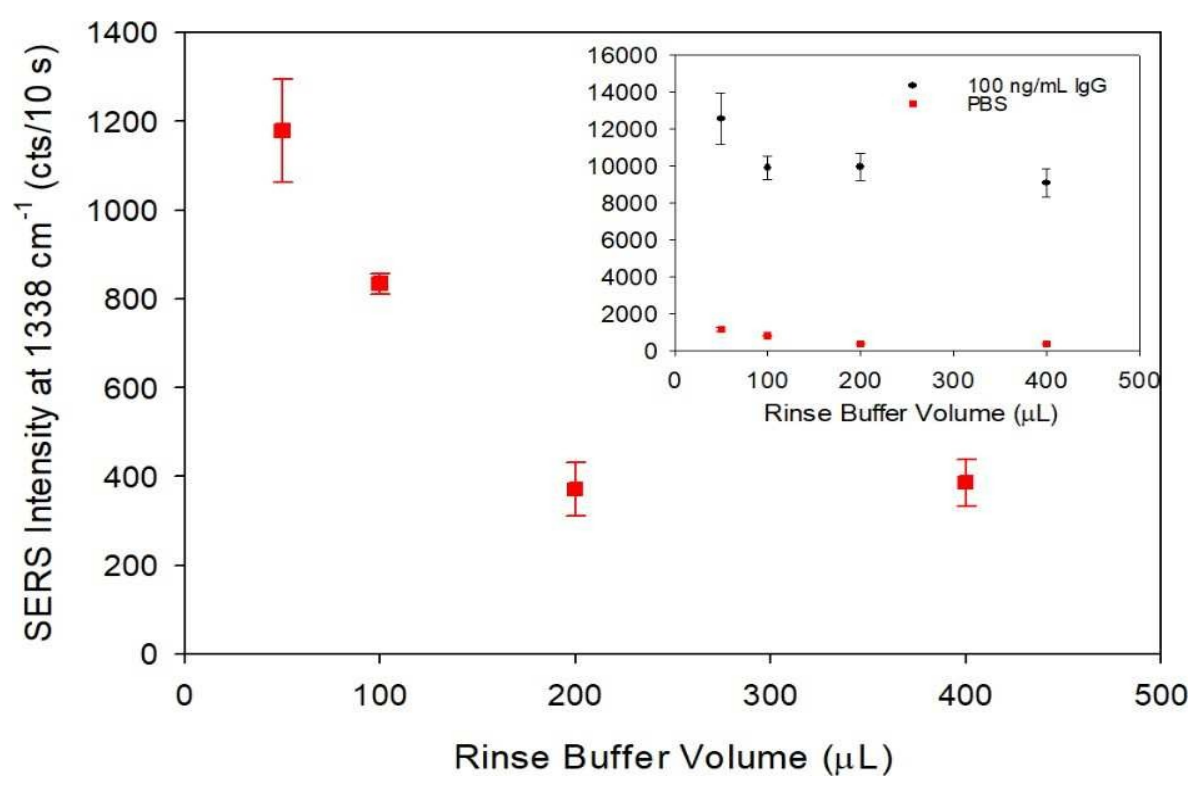

(b)

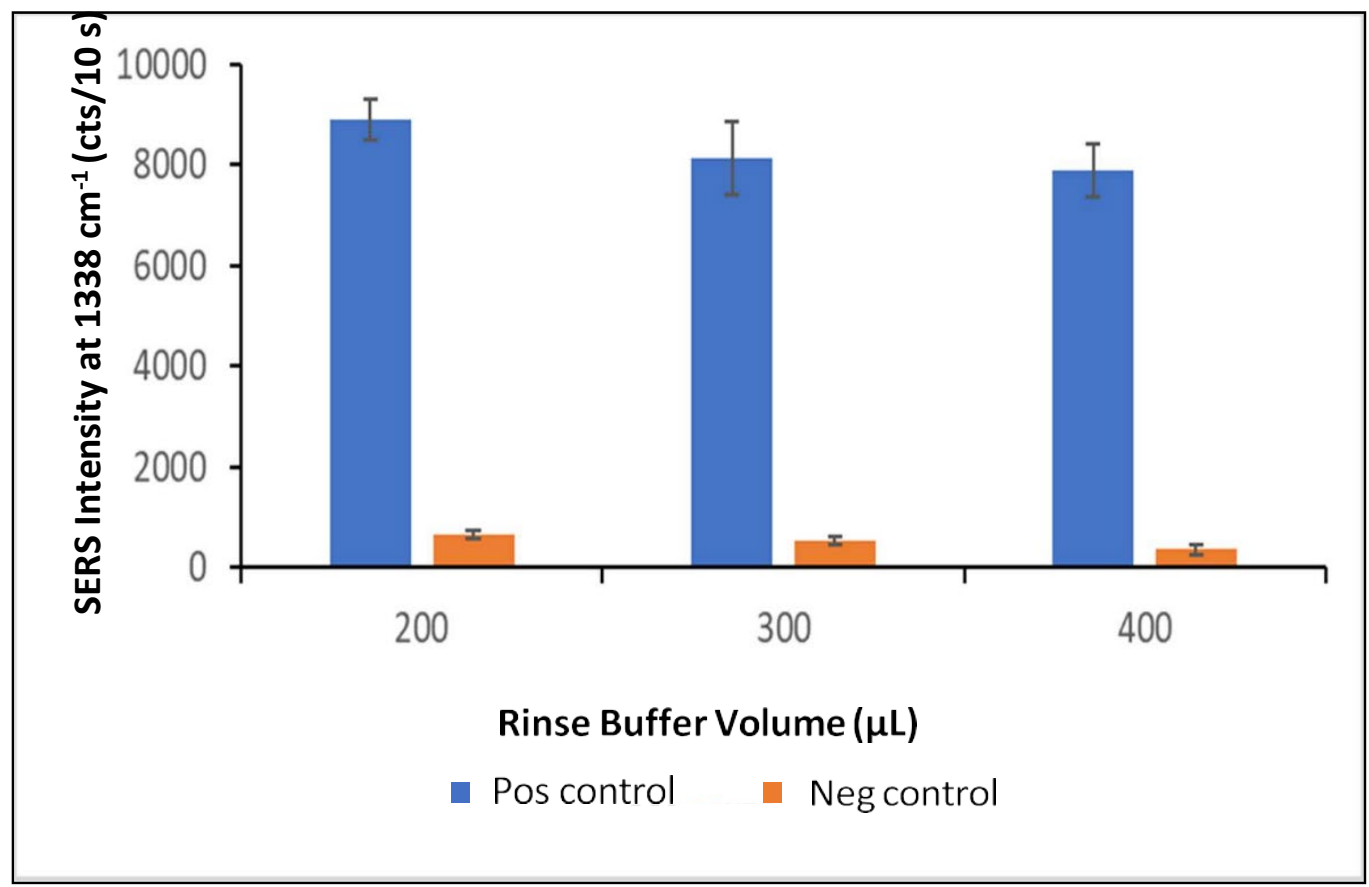

Figure 14. Effect of PBS rinsing volume on SERS intensity of capture substrate. Obtained using $2 \mu \mathrm{L}$ of $1 \mathrm{mg} / \mathrm{mL}$ anti-goat mouse $\mathrm{IgG}, 100 \mathrm{ng} / \mathrm{mL}$ of mouse IgG, $200 \mu \mathrm{L}$ ERL volumes with variable PBS rinsing volume. (a) SERS intensity as a function of rinsing buffer (b) Average SERS intensity as a function of rinsing buffer second experiment. 


\section{Analytical Performance - Dynamic Range, Detection Limit, Reproducibility,}

\section{Selectivity}

The analytical performance of the assay was evaluated using the optimized conditions to generate a calibration curve for the analysis of mouse $\operatorname{IgG}$ and establish the dynamic range, detection limit, and reproducibility. Three assays for mouse IgG were performed on three different weeks using three independently prepared suspensions of ERLs and three independently prepared sets of plasmonic paper. Raman spectra illustrating the positive and negative controls are shown in Figure 15.

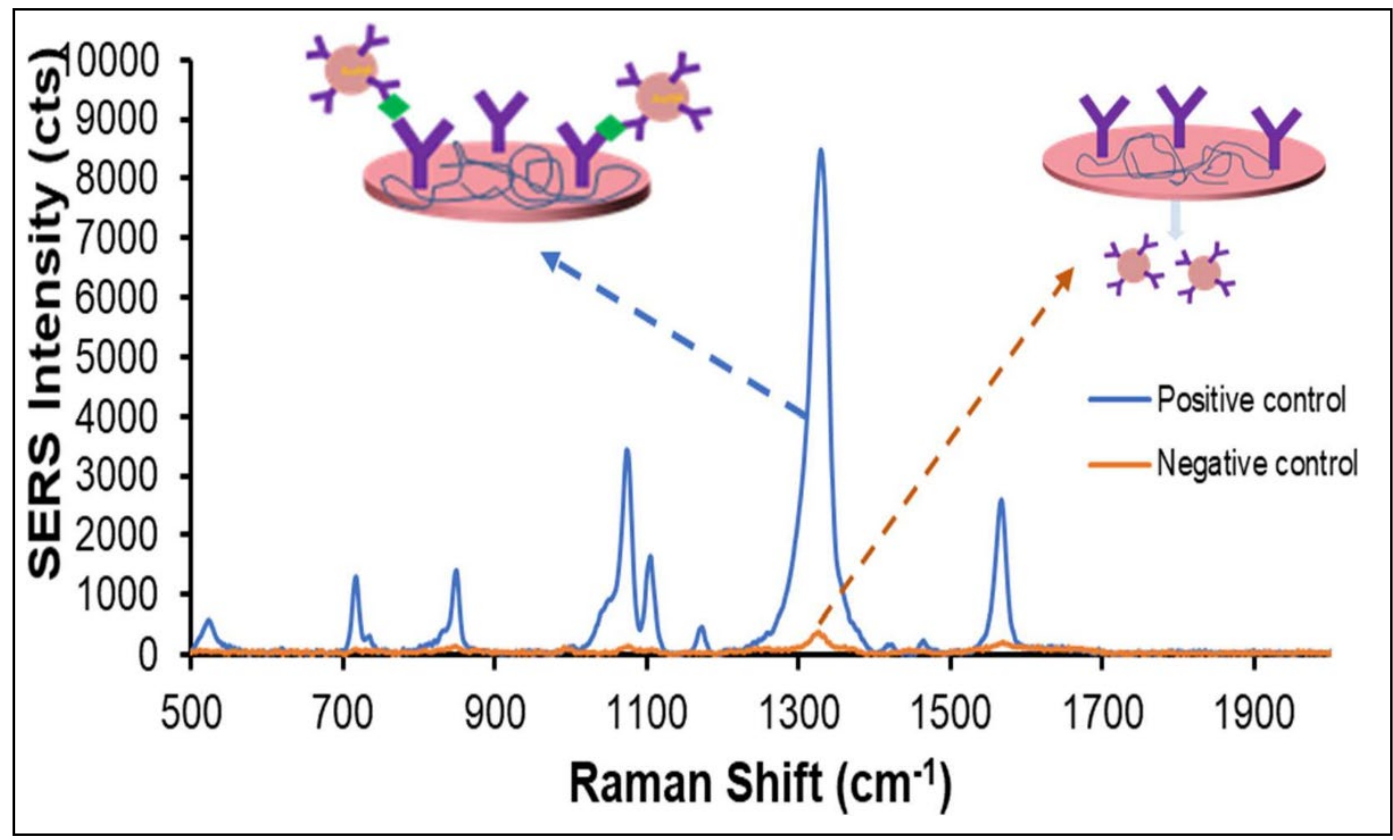

Figure 15. Illustration of SERS spectra of positive and negative control samples.

A calibration curve was generated for each independent assay to allow for analysis of intra- and inter-assay reproducibility. Concentration-dependent SERS spectra are presented in Figure 16 for one calibration dataset. 


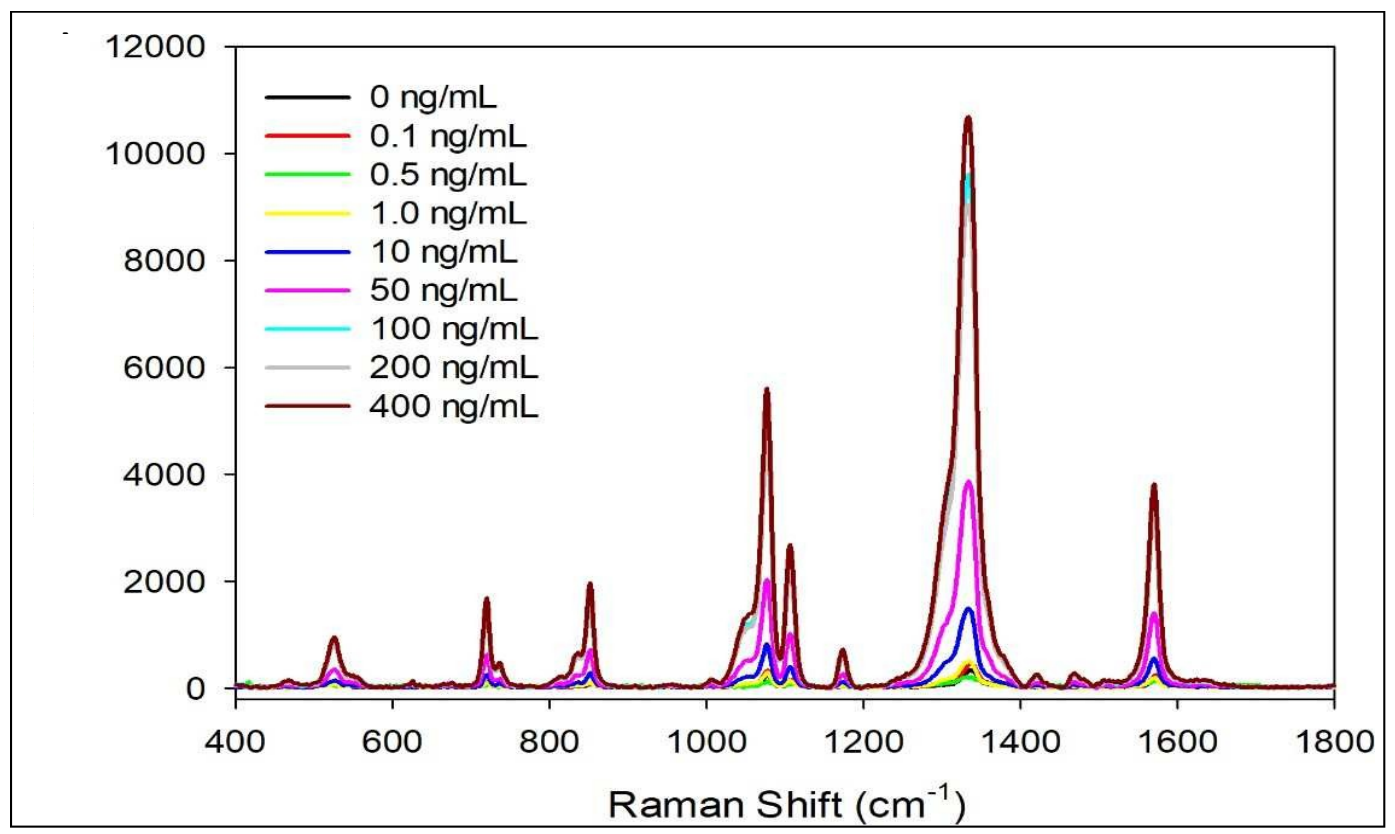

Figure 16. SERS spectra of concentration dependent assay. Obtained using optimized conditions and varying the concentration of the mouse IgG in $\mathrm{ng} / \mathrm{mL}$.

As is evident, the SERS intensity increases with a corresponding increase in antigen concentration. The most intense band at $1338 \mathrm{~cm}^{-1}$, due to the symmetric nitro stretch, is plotted as a function of antigen concentration to generate a calibration curve (Figure 17). Each data point represents the average signal collected from five different locations across the capture substrate and the error bars represent the standard deviation. The spot-to-spot variation in analytical signal measured $10-20 \%$ for most samples, although at low concentrations, e.g., $0.5 \mathrm{ng} / \mathrm{mL}$, where the signal was small, it varied as much as $37 \%$. 


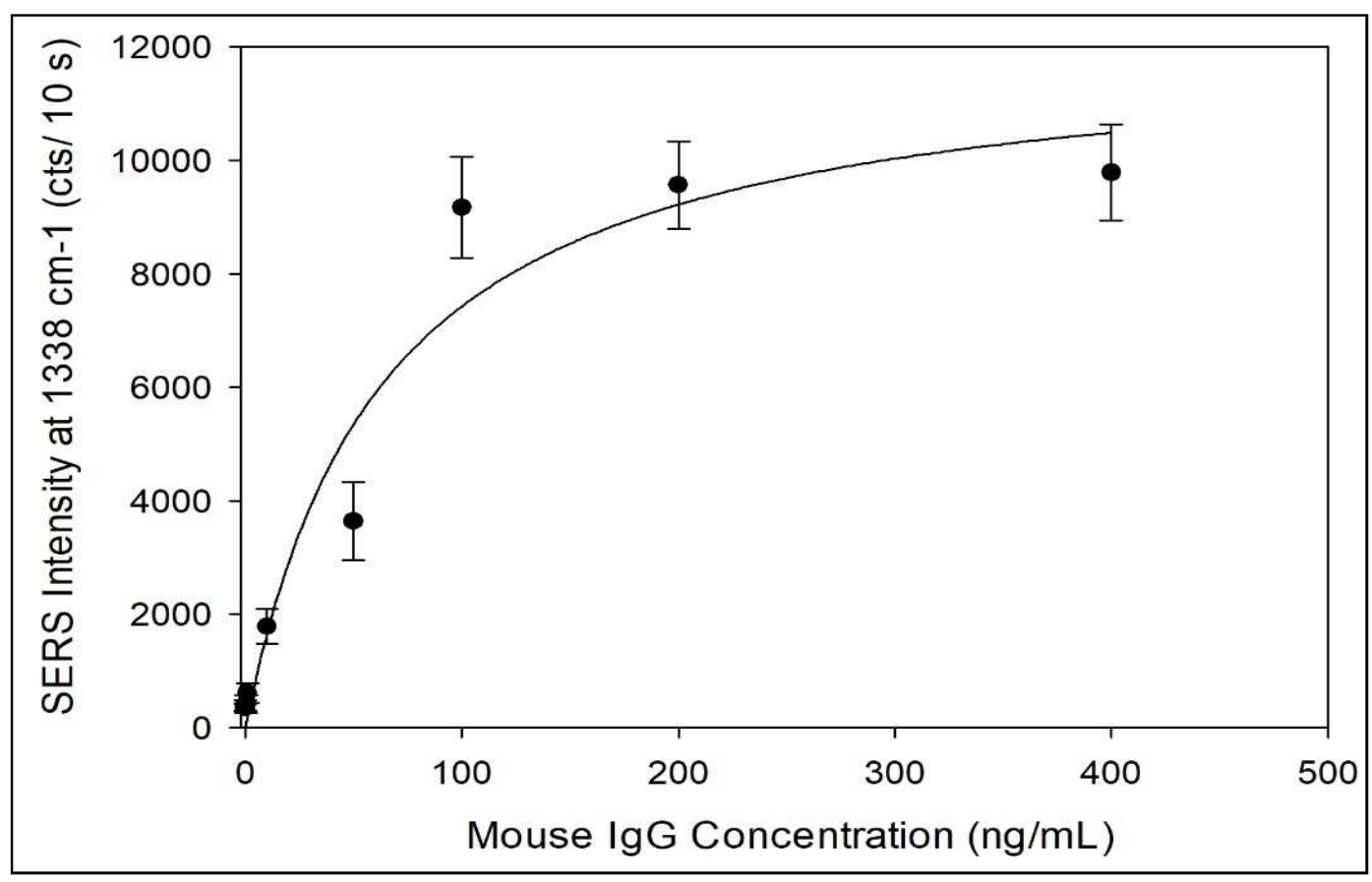

Figure 17. Calibration curve of the VFA obtained using the optimized conditions with limit of detection.

Calibration plots for two additional independent assays (assay 2 and assay 3 ) are presented in Figure 18. Critical analysis of the raw signals to evaluate the inter-assay reveals that the percent relative standard deviations (\%RSD) are 10-30\% for a given concentration. Closer inspection of the calibration plots shows that each dataset has a similar plot, in which the signal increases for sample concentrations between 0 and $100 \mathrm{ng} / \mathrm{mL}$ before reaching a maximum signal as the capture substrate becomes saturated at concentrations greater than $100 \mathrm{ng} / \mathrm{mL}$. Each of the three independent assays shows a slightly different maximum signal at saturation. While the dynamic range and overall curve shape, i.e., biding behavior, is similar for each assay, the differences in maximum signal are attributed to differences in the synthesized plasmonic paper 
substrates responsible for the SERS enhancement. To support this claim, we recently found $\sim 20 \%$ variability in SERS signals collected from plasmonic papers [98].

To account for the differences in plasmonic paper enhancement, each calibration curve was normalized with respect to its maximum signal (Figure 19). After normalization to account for substrate differences the inter-assay variability was significantly reduced to $\sim 10-20 \%$.

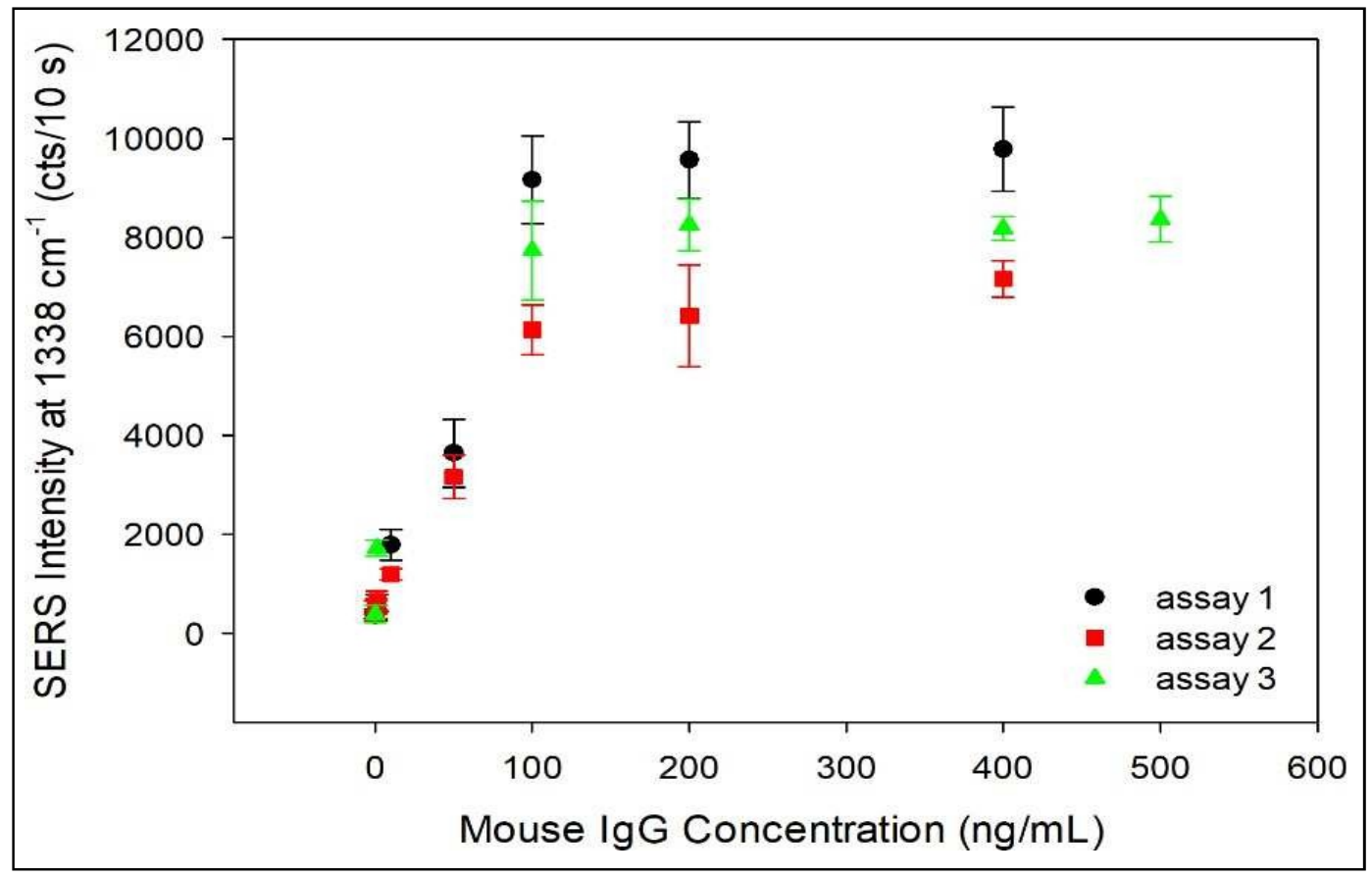

Figure 18. Calibration plots for absolute averaged intensity of three independent assays.

Obtained by plotting absolute average intensity as a function of concentration of mouse IgG in $\mathrm{ng} / \mathrm{mL}$ using the optimized condition. 


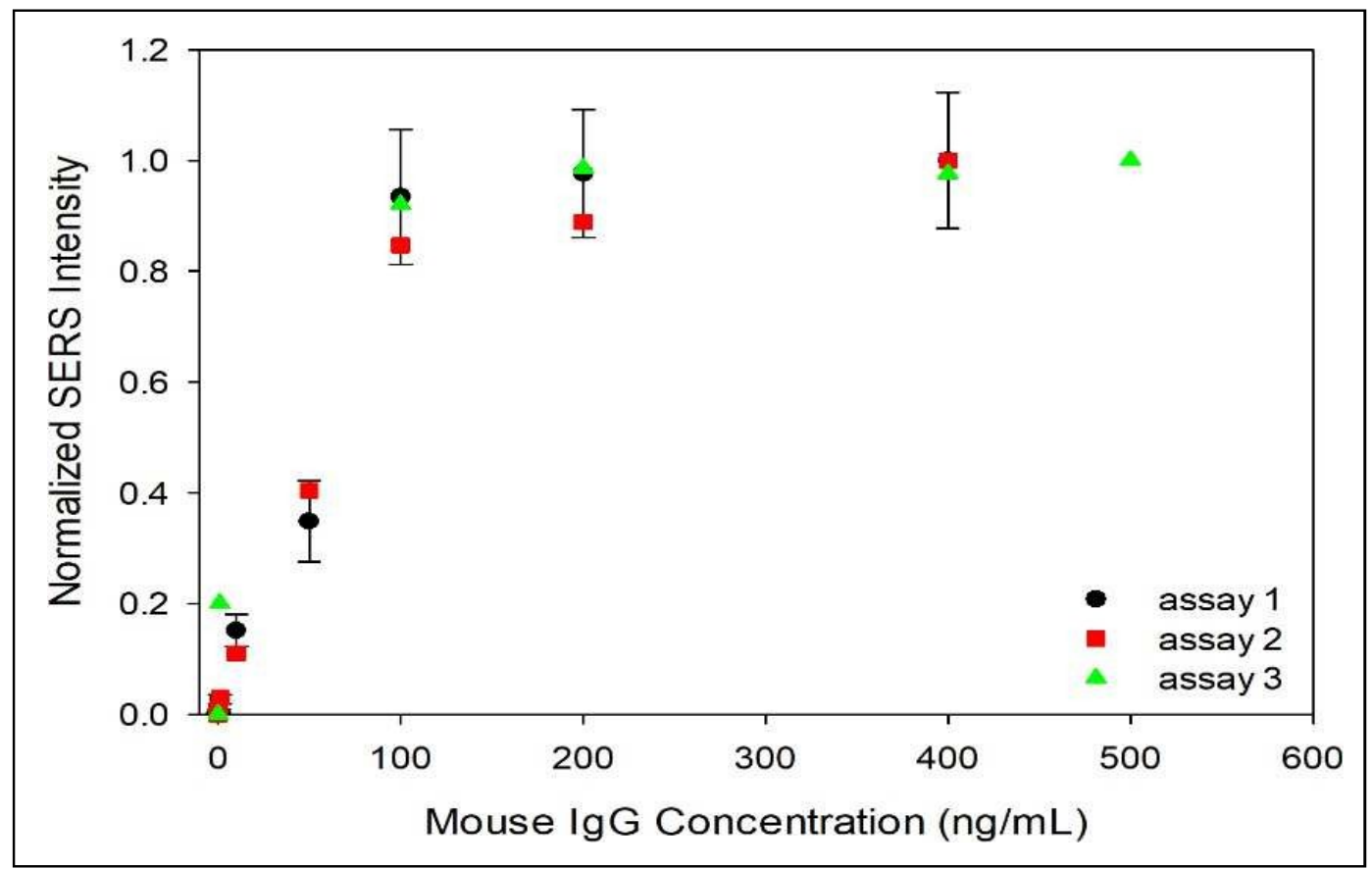

Figure 19. Calibration plots for normalized averaged intensity of three independent assays. Obtained by plotting normalized intensity as a function of concentration of mouse IgG in $\mathrm{ng} / \mathrm{mL}$ using the optimized condition.

The detection limit of the SERS vertical flow assay was defined as the antigen concentration that produces a signal equal to the blank signal plus three times the standard deviation of the blank measurement. The calibration data presented in Figure 17 was best fit to a ligand binding model, i.e., saturation curve (Equation 1) [117],

$$
y=\frac{B_{\max } x}{K_{d}+x}
$$

where $B_{\max }$ represents the maximum binding, i.e., maximum signal, and $K_{d}$ is the antibody-antigen dissociation constant. Based on the best fit of the data, and replicated signals acquired for the blank, the detection limit was $3 \mathrm{ng} / \mathrm{mL}$. Similar evaluation of the two additional 
independent calibration datasets presented in Figure 18 resulted in detection limits of 8 and 3 $\mathrm{ng} / \mathrm{mL}$. This detection limit is equivalent to that obtained for a solution based assay we previously reported using a similar antibody-antigen system, yet this current platform does not suffer from the hook effect [102]. Moreover, a filter based immunoassay using a syringe to facilitate flow rather than a vertical flow-based absorbent pad yielded a detection limit of 1-10 $\mathrm{ng} / \mathrm{mL}$ for mouse $\mathrm{IgG}$, but required $1 \mathrm{~mL}$ of sample and 10 minutes to complete the assay rather than the $100 \mu \mathrm{L}$ of sample and $\sim 2$ min assay time required for this current format [118]. It is worth noting that other vertical flow assays without a plasmonic capture substrate have reported lower detection limits than we present here $[101,119,120]$; however, those works employed monoclonal antibodies, and it is well-established that the binding affinity of the antibody-antigen governs the detection limit; thus, we can only compare to other studies that used goat anti-mouse IgG polyclonal antibodies as a model system.

In an effort to determine the effect of sample matrix on the assay performance, calibration standard solutions of mouse IgG antigen were prepared in PBS and in 10\% rabbit serum. The calibration curves generated with each of these solvents are overlaid in Figure 20. Similar calibration curves are obtained for each of these sample matrices and provide two key findings. First, these results confirm that the assay can capture the antigen protein in the presence of a complex biological matrix. Second, this experiment validates the specificity of the immunoassay given that rabbit serum contains a large concentration of rabbit IgG which is not detected by the anti-mouse IgG capture substrate and ERLs. 


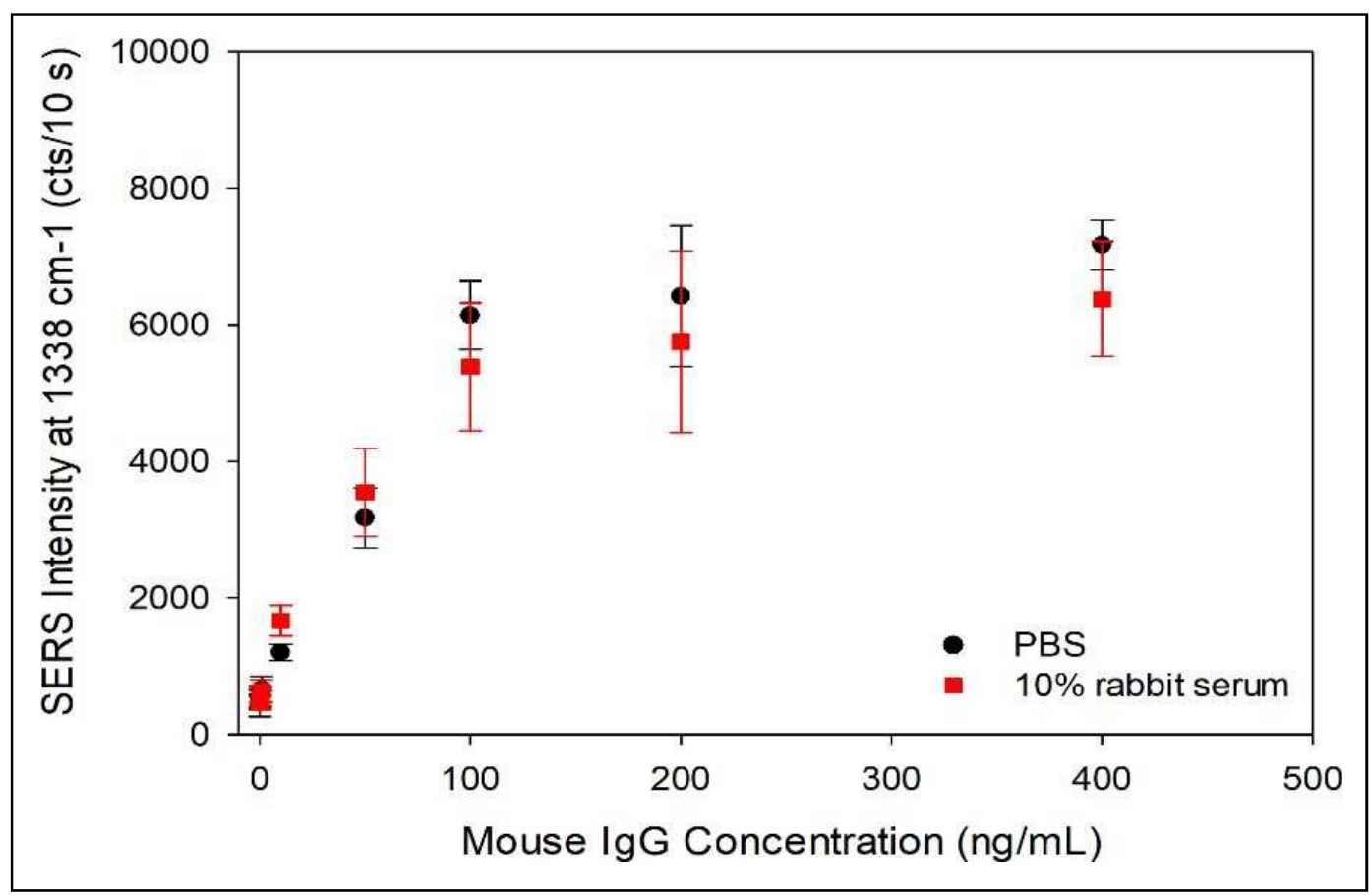

Figure 20. Calibration plots of mouse IgG prepared in PBS and 10\% rabbit serum.

\section{Application of the SERS-VFA to the Analysis of Mouse Serum}

Based on these encouraging results, we then applied our SERS-VFA to determine the concentration of mouse IgG in mouse serum. IgG is an abundant protein found in serum and has normal concentration range of $5-13 \mathrm{mg} / \mathrm{mL}$ in serum collected from healthy mice. Given that the concentration of mouse IgG in normal serum is outside of the limit of linearity (Figure 17) for the SERS-VFA, the mouse serum was diluted $1: 10^{6}$ using PBS as a diluent. We prepared three independent mouse serum samples (unknown A, B and C, Table 2) and the diluted serum samples were analyzed using a newly prepared set of plasmonic paper capture substrates. Concurrently, the capture substrates were used to analyze mouse IgG standards to generate a calibration curve. (Table 1 and Figure 16). The IgG concentration in the diluted serum measured $11 \pm 3 \mathrm{ng} / \mathrm{mL}$, based on the best fit of the calibration data to the ligand binding curve, which 
translates to an $\mathrm{IgG}$ concentration of $11 \pm 3 \mathrm{mg} / \mathrm{mL}$ in the original undiluted mouse serum sample. This experimental IgG concentration in normal mouse serum is in the expected range and is consistent with measured values for mouse $\operatorname{IgG}$ in normal serum.

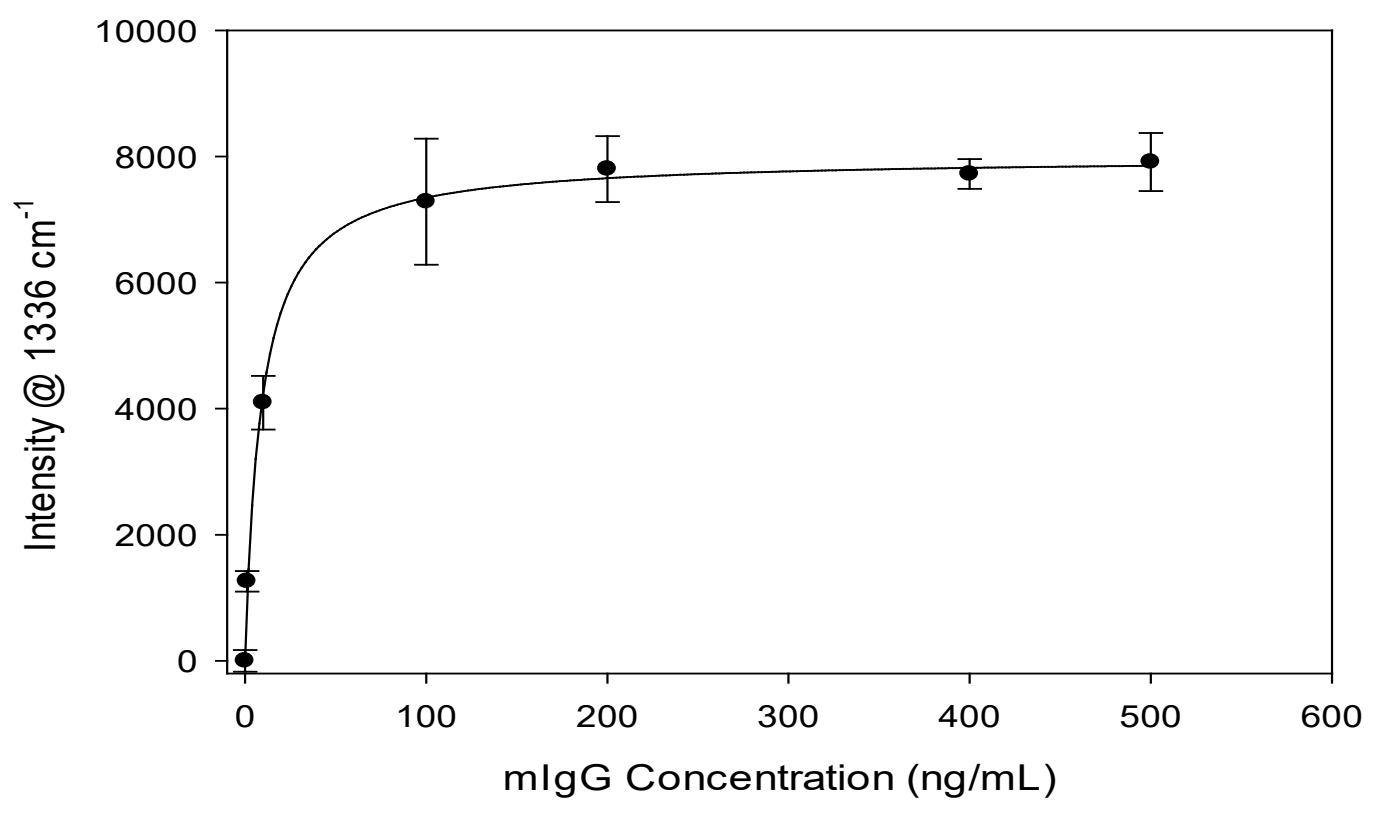

Figure 21. Calibration curve to determine IgG in mouse serum obtained using optimized condition. 


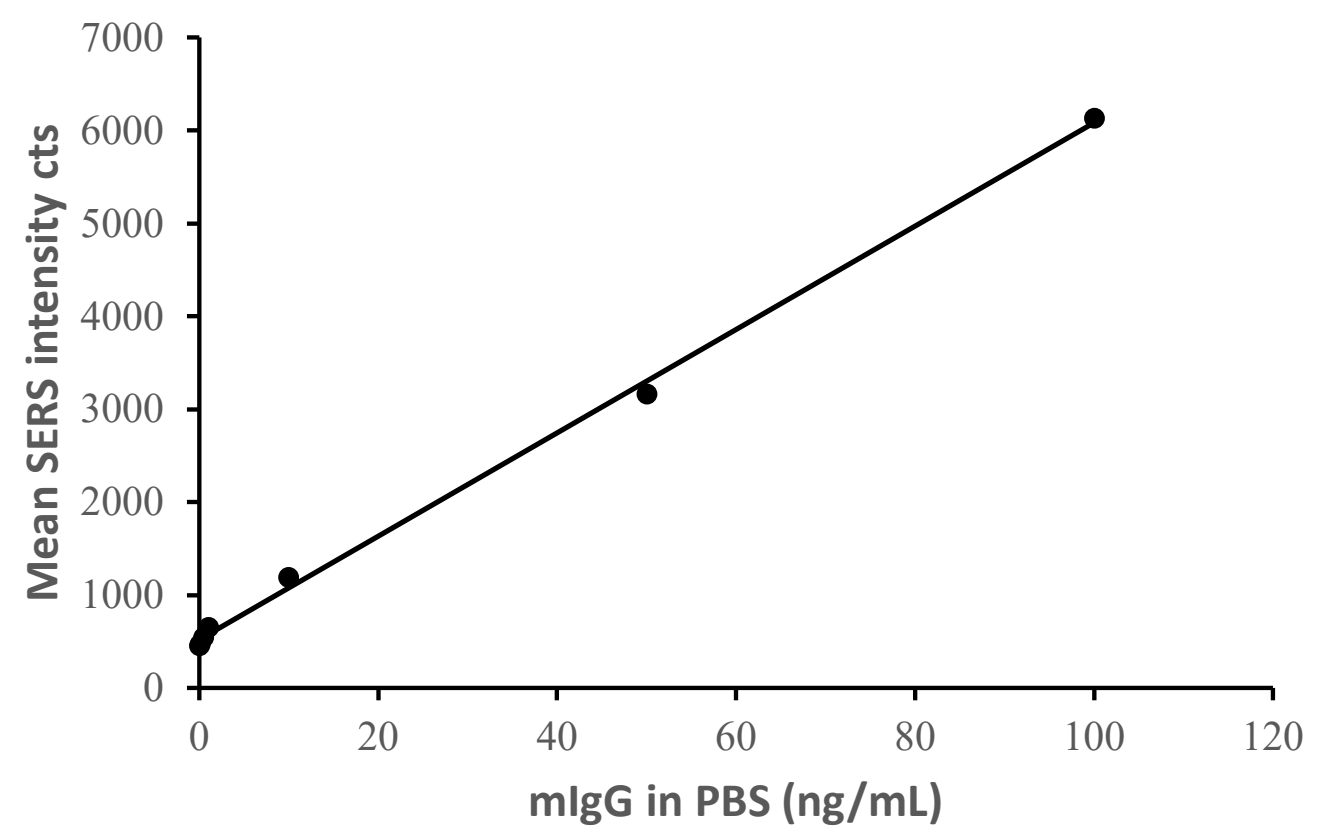

Figure 22. Limit of linearity of the calibration curve.

Table 1. Mouse IgG (mIgG) Diluted in PBS.

\begin{tabular}{cc}
\hline $\mathrm{mIgG}(\mathrm{ng} / \mathrm{mL})$ & $\begin{array}{c}\text { Mean SERS } \\
\text { Intensity (cts) }\end{array}$ \\
\hline 0 & $455 \pm 99$ \\
0.1 & $475 \pm 94$ \\
0.5 & $550 . \pm 49$ \\
1 & $655 \pm 92$ \\
10 & $1194 \pm 118$ \\
50 & $3163 \pm 241$ \\
100 & $6138 \pm 304$ \\
200 & $6417 \pm 532$ \\
400 & $7164 \pm 468$ \\
\hline
\end{tabular}


Table 2. Average SERS intensity of diluted mouse serum.

\begin{tabular}{|c|c|}
\hline $\begin{array}{l}\text { Unknown } \\
\text { (ng/mL) }\end{array}$ & $\begin{array}{r}\text { Averaged } \\
\text { SERS intensity } \\
\end{array}$ \\
\hline A & $577 \pm 62$ \\
\hline B & $683 \pm 89$ \\
\hline $\mathrm{C}$ & $1660 \pm 98$ \\
\hline
\end{tabular}




\section{CHAPTER IV: CONCLUSION AND FUTURE WORKS}

\section{Research Summary}

Over the past decades, the point-of-care diagnostic platform has become an integral part of medicine and environmental analysis due to the benefit of instant results. The current POCT that has obtained commercial success is the LFA. Despites the many advantages of these techniques, they also have limitations. Latera flow devices can produce instant results; however, they cannot be applied for an accurate quantification analysis. Moreover, LFAs suffer from a high rate of false negative results. These challenges result in inaccurate results and therefore limits its full potential in biomedical applications.

Gold nanoparticle-protein conjugation has become an active area of research due to the selective nature of antibody-based assays, biocompatibility and excellent optical properties of gold nanoparticles. There are many existing methods demonstrating various ways of immobilizing proteins on gold nanoparticles with the majority of them focused on the electronic and covalent interaction between the gold nanoparticle and proteins. Many point-of-care techniques take advantage of these to improve the current methods of detection. One area that has gained much attention is surface enhanced Raman spectroscopy (SERS) due to its unparallel sensitivity for single molecule detection. Recently, SERS-based immunoassays have become an active area of research and many researchers are coupling SERS with the current methods (e.g LFA) to improve sensitivity, quantification and analysis time.

In this work, we developed a SERS-based rapid vertical flow assay employing plasmonic paper as the capture substrate. Using mouse IgG as a model antigen, we established a novel strategy to promote a significant signal enhancement for SERS detection by AuNP-embedded filter paper compared to previously utilized paper-based substrates. Our approach takes 
advantage of the plasmonic coupling between the underlying plasmonic capture substrate paper and the nanoparticle labels, e.g., ERLs, to generate exceedingly large signals. Moreover, the vertical flow format enables the capture and concentration of analyte from a small volume of sample for rapid detection using capillary action to actively transport the sample through the capture substrate to overcome diffusion limited mass transport. Lastly, the vertical flow approach is not complicated by the hook effect for the analysis of highly concentrated samples. The ease of use, speed of analysis, low detection limits, and low cost of components suggest this assay is poised to meet the demands of point-of-care diagnostic testing, on-site environmental analysis, and point-of-need biodefense applications.

The summary of the results obtained during this study are briefly explained according to the following sections:

Section A: Optimized assay parameters

Section B: Analytical performance of the SERS-VFA

Section C: Application of the SERS vertical flow assay for determination of $\operatorname{IgG}$ concentration in mouse serum.

\section{Section A: Optimization of Assay Parameters}

Development of assay protocols for an accurate and reliable testing platform requires an optimized system that is reproducible. The first part of this research was to obtain an optimized system that enabled maximum performance of the assay. The parameters we investigated in this analysis were the effect of antibody on the capture substrate, the effect of ERL volume and the volume of the rinsing buffer. The quantities of these parameters have a significant impact on the assay results and therefore must be carefully controlled. 
As an initial step, we first optimized the amount of antibodies deposited on the plasmonic paper. The antibodies were strategically immobilized on the plasmonic papers to control the binding sites; this determined how much antibody was available on the plasmonic paper to capture the antigen upon passing the sample through the paper in order to bind to the ERL. We determined that the SERS signal response is dependent on the number of antibodies available on the surface of the paper to antigen molecules. As the number of antibodies increased the SERS signal intensity increased due to the higher number of antigens that were captured until excess antibodies were available to bound all antigen molecules.

The effect of ERL volume on SERS intensity was investigated to determine if the amount of binding, e.g ERL-antigen complex was mass dependent. The quantity of the ERLs available on the surface of the membrane determines the intensity of the SERS signal which was used to quantify amount of antigen captured from the flowing sample. Again, we determined that the volume of the ERL was directly proportional to the SERS signal intensity and that surface saturation occurs at the maximum volume where all captured antigens have become bound with the ERL.

The nonspecific bindings of ERL can result to false positive results and therefore must be minimized. This was done using PBS rinsing buffer that contains surfactants. We concluded that as the rinsing volume increases the negative control signal decreases however excess volume can marginalized the assay results and therefore must be controlled. 


\section{Section B: Analytical Performance of the SERS-VFA}

The second part of this thesis was to demonstrate the capability of our developed SERSbased vertical flow assay by determining the dynamic range, limit of detection, selectivity and reproducibility. A calibration curve was generated, and the dynamic range was established between $1-100 \mathrm{ng} / \mathrm{mL}$, while saturation occurs at greater antigen concentrations. The spot-tospot variation of the paper was found to be $\sim 10-20 \%$ which is comparable to available evidence of SERS signal collected from plasmonic paper. The calibration curve was fitted to a ligand binding curve and determined the limit of detection to be $3 \mathrm{ng} / \mathrm{mL}$ which is equivalent to a solution-based assay we previously developed using the same antigen-antibody system. In addition, our developed system uses minimal sample volume of $100 \mu \mathrm{L}$ and less time of 2 mins which is better than our previous methods which require $1 \mathrm{~mL}$ of sample solution and 10 mins for analysis. We were able to establish the selectivity of our system by generating a calibration curve using mouse IgG prepared in $10 \%$ rabbit serum which is similar to that prepared in the PBS.

\section{Section C: Application of the SERS-VFA to Determine IgG in Mouse Serum}

Our vertical flow assay was used to investigate the concentration of mouse IgG in normal mouse serum to evaluate the quantitative capability of the technique. The mouse serum which has high concentration of mouse $\operatorname{IgG}(5-13 \mathrm{mg} / \mathrm{mL})$ falls outside the limit of linearity of our SERS VFA. By a million times dilution of the serum followed by SERS analysis the amount of mouse IgG present in the serum was accurately determined. 


\section{Future Works}

One avenue of future work will include extending our current SERS-based detection strategy to applications involving simultaneous multiplexed detection. The use of ERLs prepared using different antibodies and Raman labels allowed simultaneous detection of different biomarkers by first immobilizing two different types of capture antibodies on the plasmonic paper. A sample with the potential to contain two antigens would then pass through the plasmonic capture substrate and each antigen would be extracted by its respective antibody. The mixed ERLS would then be used to label captured antigens. The unique SERS spectra of each Raman label would then be used to identify the presence and quantity of each antigen independently.

A second avenue worthy of exploration is the plasmonic system. In this SERS RVF assay, spherical gold nanoparticles are embedded in the paper substrate and spherical goldserves as the core of the ERL. We have recently shown that sandwiched structures of different plasmonic nanoparticles can lead to better plasmonic coupling and SERS enhancement than the AuNP-AuNP system used to develop this assay. In this work miRNA was sandwiched between a silver nanoparticle and anisotropic gold nanoparticle. As is evident in ref [61], SERS response of the miRNA sandwiched between a silver nanoparticle and anisotropic gold nanoparticle was substantially greater than miRNA sandwiched between two spherical AuNPs. These results suggest our plasmonic capture substrate should be prepared with anisotropic gold particles and the ERLs should consist of a silver nanoparticle core for even greater enhancement. In addition, exploring different plasmonic particles, we also plan to apply a third layer of these different types of nanostructures to potentially increase the sensitivity of the SERS-VFA. In our preliminary work we analyzed samples by applying a third layer of concave cube solution of 
AuNP to a sandwiched mouse IgG between two spherical AuNPs. The SERS response in the third layer analysis shows greater enhancement than that between the sandwiched system as evidenced in Figure 18. This result also demonstrates the capability of the concave cube gold nanoparticle to be used as ERL and plasmonic paper to increase the sensitivity of the SERSVFA.

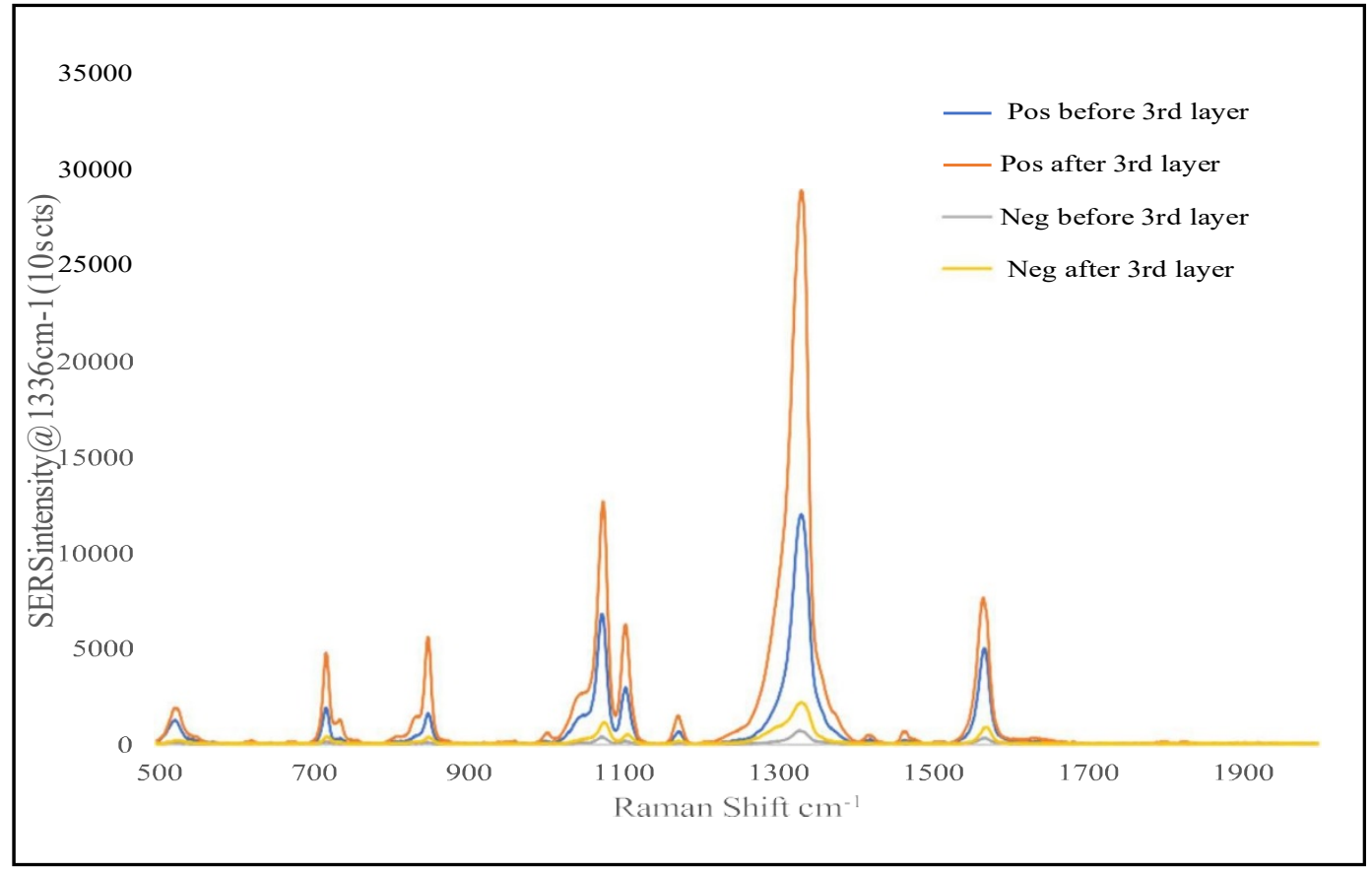

Figure 23. SERS spectra of concave cube AuNPs solution 3rd layer analysis of mouse IgG. Obtained using $10 \mu \mathrm{g} / \mathrm{ml}$ mouse IgG with the optimized conditions of the SERS-VFA. 


\section{REFERENCES}

1. Maher, D., N., Ford, N., Unwin, N. Priorities for developing countries in the global response to non-communicable diseases. Globalization and health, 2012. 8: p. 14.

2. Gubala, V., L.F. Harris, A.J. Ricco, M.X. Tan, and D.E. Williams, Point of care diagnostics: status and future. Anal Chem, 2012. 84(2): p. 487-515.

3. Gubala, V., L.F. Harris, A.J. Ricco, M.X. Tan, and D.E. Williams, Point of care diagnostics: status and future. Analytical Chemistry, 2012. 84(2): p. 487-515.

4. Kilgore, M.L., S.J. Steindel, and J.A. Smith, Evaluating stat testing options in an academic health center: therapeutic turnaround time and staff satisfaction. Clinical Chemistry, 1998. 44(8): p. 1597-1603.

5. Luppa, P. B., Müller, C., Schlichtiger, A., \& Schlebusch, H. (2011). Point-of-care testing (POCT): Current techniques and future perspectives. 2011. 30(6): p. 887-898.

6. Markets, M.A. Point of care diagnostics market. 2019; Available from: https://www.marketsandmarkets.com/Market-Reports/point-of-care-diagnostic-market106829185.html.

7. Wire, B., Global Point-of-Care Testing Market - Focus on Application, Market Share, Product Mapping and Country - Analysis and Forecast (2017-2026). Research and markets, 2018.

8. Vashist, S.K., Point-of-Care Diagnostics: Recent Advances and Trends. Biosensors Journal, 2017. 7(4). p. 62-69.

9. Li, Y., X. Yan, X. Feng, J. Wang, W. Du, Y. Wang, P. Chen, L. Xiong, and B.-F. Liu, Agarose-Based Microfluidic Device for Point-of-Care Concentration and Detection of Pathogen. Analytical Chemistry, 2014. 86(21): p. 10653-10659. 
10. Wu, H., Z. Ma, C. Wei, M. Jiang, X. Hong, Y. Li, D. Chen, and X. Huang, ThreeDimensional Microporous Hollow Fiber Membrane Microfluidic Device Integrated with Selective Separation and Capillary Self-Driven for Point-of-Care Testing. Analytical Chemistry, 2020. 92(9): p. 6358-6365.

11. Miesler, T., C. Wimschneider, A. Brem, and L. Meinel, Frugal Innovation for Point-ofCare Diagnostics Controlling Outbreaks and Epidemics. ACS Biomaterials Science \& Engineering, 2020. 6(5): p. 2709-2725.

12. Guler, E., T. Yilmaz Sengel, Z.P. Gumus, M. Arslan, H. Coskunol, S. Timur, andY. Yagci, Mobile Phone Sensing of Cocaine in a Lateral Flow Assay Combined with a Biomimetic Material. Analytical Chemistry, 2017. 89(18): p. 9629-9632.

13. Paudel, M.K., O. Shirota, K. Sasaki-Tabata, H. Tanaka, S. Sekita, and S. Morimoto, Development of an Enzyme Immunoassay Using a Monoclonal Antibody against the Psychoactive Diterpenoid Salvinorin A. Journal of Natural Products, 2013. 76(9): p. 1654-1660.

14. Wang, Z., S. Zong, L. Wu, D. Zhu, and Y. Cui, SERS-Activated Platforms for Immunoassay: Probes, Encoding Methods, and Applications. Chemical Reviews, 2017. 117(12): p. 7910-7963.

15. Wild, D., The Immunoassay Handbook, 4th Edition. Theory and Applications of Ligand Binding, ELISA and Related Techniques. 2013: Elsevier Science. p. 7-29.

16. Basilicata, P., M. Pieri, V. Settembre, A. Galdiero, E. Della Casa, A. Acampora, and N. Miraglia, Screening of Several Drugs of Abuse in Italian Workplace Drug Testing: Performance Comparisons of On-Site Screening Tests and a Fluorescence Polarization Immunoassay-Based Device. Analytical Chemistry, 2011. 83(22): p. 8566-8574. 
17. Clarke, W., J.E. Schiel, A. Moser, and D.S. Hage, Analysis of Free Hormone Fractions by an Ultrafast Immunoextraction/Displacement Immunoassay: Studies Using Free Thyroxine as a Model System. Analytical Chemistry, 2005. 77(6): p. 1859-1866.

18. Wang, R., H. Chon, S. Lee, Z. Cheng, S.H. Hong, Y.H. Yoon, and J. Choo, Highly Sensitive Detection of Hormone Estradiol E2 Using Surface-Enhanced Raman Scattering Based Immunoassays for the Clinical Diagnosis of Precocious Puberty. ACS Applied Materials \& Interfaces, 2016. 8(17): p. 10665-10672.

19. Driskell, J.D., K.M. Kwarta, R.J. Lipert, M.D. Porter, J.D. Neill, and J.F. Ridpath, LowLevel Detection of Viral Pathogens by a Surface-Enhanced Raman Scattering Based Immunoassay. Analytical Chemistry, 2005. 77(19): p. 6147-6154.

20. Wu, Z., T. Zeng, W.-J. Guo, Y.-Y. Bai, D.-W. Pang, and Z.-L. Zhang, Digital Single Virus Immunoassay for Ultrasensitive Multiplex Avian Influenza Virus Detection Based on Fluorescent Magnetic Multifunctional Nanospheres. ACS Applied Materials \& Interfaces, 2019. 11(6): p. 5762-5770.

21. Aga, D.S., Thurman E.M. Environmental Immunoassays: Alternative Techniques for Soil and Water Analysis, in Immunochemical Technology for Environmental Applications. 1997, American Chemical Society. p. 1-20.

22. Cochrane, W.P., Testing of Food and Agricultural Products by Immunoassay, in Immunoassays or Trace Chemical Analysis. 1990, American Chemical Society. p. 40-48.

23. Lu, C.M., D.W. Burton, R.L. Fitzgerald, L.J. Deftos, B.A. Buchholz, J.S. Vogel, and D.A. Herold, Mass Spectrometric Immunoassay for Parathyroid Hormone-Related Protein. Analytical Chemistry, 2002. 74(21): p. 5507-5512. 
24. Yan, X., W. Zhong, A. Tang, E.G. Schielke, W. Hang, and J.P. Nolan, Multiplexed Flow Cytometric Immunoassay for Influenza Virus Detection and Differentiation. Analytical Chemistry, 2005. 77(23): p. 7673-7678.

25. Manz, A., Bioanalytical chemistry. 2nd ed, ed. P.S. Dittrich, N. Pamme, and D. Iossifidis. 2004, London: Imperial college press. 236-249.

26. Bhimji, A., A.A. Zaragoza, L.S. Live, and S.O. Kelley, ElectrochemicalEnzyme-Linked Immunosorbent Assay Featuring Proximal Reagent Generation: Detection of Human Immunodeficiency Virus Antibodies in Clinical Samples. Analytical Chemistry, 2013. 85(14): p. 6813-6819.

27. Sevenler, D., A. Bardon, M. Fernandez Suarez, L. Marshall, M. Toner, P.K. Drain, and R.D. Sandlin, Immunoassay for HIV Drug Metabolites Tenofovir and Tenofovir Diphosphate. ACS Infectious Diseases, 2020.

28. Miodek, A., J. Vidic, H. Sauriat-Dorizon, C.-A. Richard, R. Le Goffic, H. KorriYoussoufi, and C. Chevalier, Electrochemical Detection of the Oligomerization of PB1F2 Influenza A Virus Protein in Infected Cells. Analytical Chemistry, 2014. 86(18): p. 9098-9105.

29. Kalkal, A., R. Pradhan, S. Kadian, G. Manik, and G. Packirisamy, Biofunctionalized graphene quantum dots based fluorescent biosensor towards efficient detection of small cell lung cancer. ACS Applied Bio Materials, 2020.

30. Fu, K., Y. Zheng, J. Li, Y. Liu, B. Pang, X. Song, K. Xu, J. Wang, and C. Zhao, Colorimetric Immunoassay for Rapid Detection of Vibrio parahemolyticus Based on Mn2+ Mediates the Assembly of Gold Nanoparticles. Journal of Agricultural andFood Chemistry, 2018. 66(36): p. 9516-9521. 
31. Matabaro, E., N. Ishimwe, Eric Uwimbabazi, and B.H. Lee, Current immunoassay methods for the rapid detection of aflatoxin in milk and dairy products. Comprehensive Reviews in Food Science and Food Safety, 2017. 16. p. 809 - 817.

32. Wang, Z., S. Zhang, I.S. Nesterenko, S.A. Eremin, and J. Shen, Monoclonal AntibodyBased Fluorescence Polarization Immunoassay for Sulfamethoxypyridazine and Sulfachloropyridazine. Journal of Agricultural and Food Chemistry, 2007. 55(17): p. 6871-6878.

33. Liu, W., C.L. Cassano, X. Xu, and Z.H. Fan, Laminated Paper-Based Analytical Devices (LPAD) with Origami-Enabled Chemiluminescence Immunoassay for Cotinine Detection in Mouse Serum. Analytical Chemistry, 2013. 85(21): p. 10270-10276.

34. Tang, T.-C., A. Deng, and H.-J. Huang, Immunoassay with a Microtiter Plate Incorporated Multichannel Electrochemical Detection System. Analytical Chemistry, 2002. 74(11): p. 2617-2621.

35. Wang, G., R.J. Lipert, M. Jain, S. Kaur, S. Chakraboty, M.P. Torres, S.K. Batra, R.E. Brand, and M.D. Porter, Detection of the potential pancreatic cancer marker MUC4 in serum using surface-enhanced Raman scattering. Anal Chem, 2011. 83(7): p. 2554-61.

36. Shan, S., W. Lai, Y. Xiong, H. Wei, and H. Xu, Novel Strategies To Enhance Lateral Flow Immunoassay Sensitivity for Detecting Foodborne Pathogens. Journal of Agricultural and Food Chemistry, 2015. 63(3): p. 745-753.

37. Ji, P., J. Zhu, X. Li, W. Fan, Q. Liu, K. Wang, J. Zhao, Y. Sun, B. Liu, E.-M. Zhou, and Q. Zhao, Fenobody and RANbody-based sandwich enzyme-linked immunosorbent assay to detect Newcastle disease virus. Journal of Nanobiotechnology, 2020. 18(1): p. 2-19. 
38. Lee, L.G., E.S. Nordman, M.D. Johnson, and M.F. Oldham, A low-cost, highperformance system for fluorescence lateral flow assays. Biosensors (Basel), 2013. 3(4): p. $360-73$.

39. Zhang, Y., X. Liu, L. Wang, H. Yang, X. Zhang, C. Zhu, W. Wang, L. Yan, and B. Li, Improvement in Detection Limit for Lateral Flow Assay of Biomacromolecules by TestZone Pre-enrichment. Scientific Reports, 2020. 10(1): p. 9604.

40. Hansen, E.S. and J. Knudsen, Limitations in the use of the enzyme-linked immunosorbent assay (ELISA) for identification and quantification of thermogenin. J Immunol Methods, 1985. 77(2): p. 297-304.

41. Gomez-Martinez, J., M. Silvy, J. Chiaroni, C. Fournier-Wirth, F. Roubinet, P. Bailly, and J.-C. Brès, Multiplex Lateral Flow Assay for Rapid Visual Blood Group Genotyping. Analytical Chemistry, 2018. 90(12): p. 7502-7509.

42. Hamad, E.M., G. Hawamdeh, N.A. Jarrad, O. Yasin, S.I. Al-Gharabli, and R. Shadfan, Detection of Human Chorionic Gonadotropin (hCG) Hormone using Digital Lateral Flow Immunoassay. Conf Proc IEEE Eng Med Biol Soc, 2018. 2018: p. 3845-3848.

43. Mahmoudi, T., de la Guardia M., Baradaran., B. Lateral flow assays towards point -of care cancer detection: A review of current progress and future trends. Trac-Trends in Analytical Chemistry, 2020. 125. p. 1096-1106

44. Rohrman, B.A., V. Leautaud, E. Molyneux, and R.R. Richards-Kortum, A Lateral Flow Assay for Quantitative Detection of Amplified HIV-1 RNA. Plos One, 2012. 7(9).p.1-7.

45. Fu, X., Z. Cheng, J. Yu, P. Choo, L. Chen, and J. Choo, A SERS-based lateral flow assay biosensor for highly sensitive detection of HIV-1 DNA. Biosens Bioelectron, 2016. 78: p. $530-537$. 
46. Xu, Y., Y. Liu, Y. Wu, X. Xia, Y. Liao, and Q. Li, Fluorescent Probe-Based Lateral Flow Assay for Multiplex Nucleic Acid Detection. Analytical Chemistry, 2014. 86(12): p. 5611-5614.

47. Wang, X., N. Choi, Z. Cheng, J. Ko, L. Chen, and J. Choo, Simultaneous Detection of Dual Nucleic Acids Using a SERS-Based Lateral Flow Assay Biosensor. Analytical Chemistry, 2017. 89(2): p. 1163-1169.

48. Posthuma-Trumpie, G.A., J. Korf, and A. van Amerongen, Lateral flow (immuno)assay: its strengths, weaknesses, opportunities and threats. A literature survey. Anal Bioanal Chem, 2009. 393(2): p. 569-82.

49. Hurt, A.C., R. Alexander, J. Hibbert, N. Deed, and I.G. Barr, Performance of six influenza rapid tests in detecting human influenza in clinical specimens. J Clin Virol, 2007. 39(2): p. 132-235.

50. Chen, P., M. Gates-Hollingsworth, S. Pandit, A. Park, D. Montgomery, D. AuCoin, J. $\mathrm{Gu}$, and F. Zenhausern, Paper-based Vertical Flow Immunoassay (VFI) for detection of bio-threat pathogens. Talanta, 2019. 191: p. 81-88.

51. Clarke, O.J., B.L. Goodall, H.P. Hui, N. Vats, and C.L. Brosseau, Development of a SERS-Based Rapid Vertical Flow Assay for Point-of-Care Diagnostics. Anal Chem, 2017. 89(3): p. 1405-1410.

52. Frasconi, M., C. Tortolini, F. Botrè, and F. Mazzei, Multifunctional Au Nanoparticle Dendrimer-Based Surface Plasmon Resonance Biosensor and Its Application for Improved Insulin Detection. Analytical Chemistry, 2010. 82(17): p. 7335-7342. 
53. Chen, W., L. Zheng, M. Wang, Y. Chi, and G. Chen, Preparation of Protein-like SilverCysteine Hybrid Nanowires and Application in Ultrasensitive Immunoassay of Cancer Biomarker. Analytical Chemistry, 2013. 85(20): p. 9655-9663.

54. Ranzoni, A., A. den Hamer, T. Karoli, J. Buechler, and M.A. Cooper, Improved Immunoassay Sensitivity in Serum as a Result of Polymer-Entrapped Quantum Dots: 'Papaya Particles'. Analytical Chemistry, 2015. 87(12): p. 6150-6157.

55. Chen, N., P. Ding, Y. Shi, T. Jin, Y. Su, H. Wang, and Y. He, Portable and Reliable Surface-Enhanced Raman Scattering Silicon Chip for Signal-On Detection of Trace Trinitrotoluene Explosive in Real Systems. Analytical Chemistry, 2017. 89(9): p. 50725078.

56. Brewster, V.L., L. Ashton, R. Goodacre, Monitoring the Glycosylation Status of Proteins Using Raman Spectroscopy. Analytical Chemistry, 2011. 83(15): p. 6074-6081.

57. Okotrub, K.A., N.V. Surovtsev, V.F. Semeshin, and L.V. Omelyanchuk, Raman spectroscopy for DNA quantification in cell nucleus. Cytometry A, 2015. 87(1): p.68-73.

58. Ojeda, J.F., C. Xie, Y.Q. Li, F.E. Bertrand, J. Wiley, and T.J. McConnell, Chromosomal analysis and identification based on optical tweezers and Raman spectroscopy: reply. Opt Express, 2007. 15(10): p. 6000-6012.

59. Shashni, B., Y. Horiguchi, K. Kurosu, H. Furusho, and Y. Nagasaki, Application of surface enhanced Raman spectroscopy as a diagnostic system for hypersialylated metastatic cancers. Biomaterials, 2017. 134: p. 143-153.

60. Ghosh, S.K. T. Pal, Interparticle Coupling Effect on the Surface Plasmon Resonance of Gold Nanoparticles: From Theory to Applications. Chemical Reviews, 2007. 107(11): p. 4797-4862. 
61. Lartey, J.A., Harms, J.P., Frimpong, R., Mulligan, C.C., Driskell, J.D., Kim, J.-H., Sandwiching analytes with structurally diverse plasmonic nanoparticles on paper substrates for surface enhanced Raman spectroscopy. RSC Advances, 2019. 9(56): p. $32535-32543$.

62. Lee, H.K., Y.H. Lee, C.S.L. Koh, G.C. Phan-Quang, X. Han, C.L. Lay, H.Y.F. Sim, Y.C. Kao, Q. An, and X.Y. Ling, Designing surface-enhanced Raman scattering (SERS) platforms beyond hotspot engineering: emerging opportunities in analyte manipulations and hybrid materials. Chemical Society Reviews, 2019. 48(3): p. 731-756.

63. Wang, Z., S. Zong, L. Wu, D. Zhu, and Y. Cui, SERS-Activated Platforms for Immunoassay: Probes, Encoding Methods, and Applications. Chem Rev, 2017. 117(12): p. $7910-7963$.

64. Hall, W.P., S.N. Ngatia, R.P. Van Duyne, LSPR Biosensor Signal Enhancement Using Nanoparticle-Antibody Conjugates. J Phys Chem C Nanomater Interfaces, 2011.115(5): p. $1410-1414$.

65. Qian, X., J. Li, S. Nie, Stimuli-Responsive SERS Nanoparticles: Conformational Control of Plasmonic Coupling and Surface Raman Enhancement. Journal of the American Chemical Society, 2009. 131(22): p. 7540-7541.

66. Kang, H., J.T. Buchman, R.S. Rodriguez, H.L. Ring, J. He, K.C. Bantz, and C.L.Haynes, Stabilization of Silver and Gold Nanoparticles: Preservation and Improvement of Plasmonic Functionalities. Chemical Reviews, 2019. 119(1): p. 664-699.

67. Wang, Y., B. Yan, L. Chen, SERS Tags: Novel Optical Nanoprobes for Bioanalysis. Chemical Reviews, 2013. 113(3): p. 1391-1428. 
68. Altun, A.O., T. Bond, W. Pronk, and H.G. Park, Sensitive Detection of Competitive Molecular Adsorption by Surface-Enhanced Raman Spectroscopy. Langmuir, 2017. 33(28): p. 6999-7006.

69. Constantino, C.J.L., T. Lemma, P.A. Antunes, R. Aroca, Single-MoleculeDetection Using Surface-Enhanced Resonance Raman Scattering and Langmuir-Blodgett Monolayers. Analytical Chemistry, 2001. 73(15): p. 3674-3678.

70. Stranahan, S.M., E.J. Titus, and K.A. Willets, Discriminating Nanoparticle Dimers from Higher Order Aggregates through Wavelength-Dependent SERS Orientational Imaging. ACS Nano, 2012. 6(2): p. 1806-1813.

71. Zámbó, D., D.P. Szekrényes, S. Pothorszky, N. Nagy, and A. Deák, SERS Activity of Reporter-Particle-Loaded Single Plasmonic Nanovoids. The Journal of Physical Chemistry C, 2018. 122(41): p. 23683-23690.

72. Long, J., H. Yi, H. Li, Z. Lei, and T. Yang, Reproducible Ultrahigh SERSEnhancement in Single Deterministic Hotspots Using Nanosphere-Plane Antennas Under Radially Polarized Excitation. Scientific Reports, 2016. 6(1): p. 2-7.

73. Wustholz, K.L., A.I. Henry, J.M. McMahon, R.G. Freeman, N. Valley, M.E. Piotti, M.J. Natan, G.C. Schatz, and R.P. Van Duyne, Structure-activity relationships in gold nanoparticle dimers and trimers for surface-enhanced Raman spectroscopy. J Am Chem Soc, 2010. 132(31): p. $2-10$.

74. Szekeres, G.P. J. Kneipp, SERS Probing of Proteins in Gold Nanoparticle Agglomerates. Front Chem, 2019. 7: p. 2-8. 
75. Strelau, K.K., R. Kretschmer, R. Moller, W. Fritzsche, and J. Popp, SERS as tool for the analysis of DNA-chips in a microfluidic platform. Anal Bioanal Chem, 2010. 396(4): $\mathrm{p}$. $435-444$.

76. Cheng, Z., R. Wang, Y. Xing, L. Zhao, J. Choo, and F. Yu, SERS-based immunoassay using gold-patterned array chips for rapid and sensitive detection of dual cardiac biomarkers. Analyst, 2019. 144(22): p. 6533-6540.

77. Ko, J., C. Lee, J. Choo, Highly sensitive SERS-based immunoassay of aflatoxin B1 using silica-encapsulated hollow gold nanoparticles. J Hazard Mater, 2015. 285: p. 11-17.

78. Ko, J., S. Lee, E.K. Lee, S.I. Chang, L. Chen, S.Y. Yoon, and J. Choo, SERS-based immunoassay of tumor marker VEGF using DNA aptamers and silica-encapsulated hollow gold nanospheres. Phys Chem Chem Phys, 2013. 15(15): p. 5379-5385.

79. Chan, T.-Y., T.-Y. Liu, K.-S. Wang, K.-T. Tsai, Z.-X. Chen, Y.-C. Chang, Y.-Q. Tseng, C.-H. Wang, J.-K. Wang, and Y.-L. Wang, SERS Detection of Biomolecules by Highly Sensitive and Reproducible Raman-Enhancing Nanoparticle Array. Nanoscale Research Letters, 2017. 12(1): p. 12 - 344.

80. Temur, E., A. Zengin, İ.H. Boyacı, F.C. Dudak, H. Torul, and U. Tamer, Attomole Sensitivity of Staphylococcal Enterotoxin B Detection Using an Aptamer-Modified Surface-Enhanced Raman Scattering Probe. Analytical Chemistry, 2012. 84(24): p. 10600-10606.

81. Begandt, D., A. Bader, G.C. Antonopoulos, M. Schomaker, S. Kalies, H. Meyer, T. Ripken, and A. Ngezahayo, Gold nanoparticle-mediated (GNOME) laser perforation: a new method for a high-throughput analysis of gap junction intercellular coupling. $\mathrm{J}$ Bioenerg Biomembr, 2015. 47(5): p. 441-449. 
82. Wustholz, K.L., A.-I. Henry, J.M. McMahon, R.G. Freeman, N. Valley, M.E. Piotti, M.J. Natan, G.C. Schatz, and R.P. Van Duyne, Structure-Activity Relationships in Gold Nanoparticle Dimers and Trimers for Surface-Enhanced Raman Spectroscopy. Journal of the American Chemical Society, 2010. 132(31): p. 10903-10910.

83. Guerrini, L., F. McKenzie, A.W. Wark, K. Faulds, and D. Graham, Tuning the interparticle distance in nanoparticle assemblies in suspension via DNA-triplex formation: correlation between plasmonic and surface-enhanced Raman scattering responses. Chemical Science, 2012. 3(7): p. 2262-2269.

84. Cui, Y., H. Wang, S. Liu, Y. Wang, and J. Huang, Target-activated DNA nanomachines for the ATP detection based on the SERS of plasmonic coupling from gold nanoparticle aggregation. Analyst, 2020. 145(2): p. 445-452.

85. Clarke, O.J.R., B.L. Goodall, H.P. Hui, N. Vats, and C.L. Brosseau, Development of a SERS-Based Rapid Vertical Flow Assay for Point-of-Care Diagnostics. Analytical Chemistry, 2017. 89(3): p. 1405-1410.

86. Li, X., Y. Zhang, B. Xue, X. Kong, X. Liu, L. Tu, Y. Chang, and H. Zhang, A SERS nano-tag-based fiber-optic strategy for in situ immunoassay in unprocessed whole blood. Biosens Bioelectron, 2017. 92: p. 517-522.

87. Penn, M.A., D.M. Drake, J.D. Driskell, Accelerated Surface-Enhanced Raman Spectroscopy (SERS)-Based Immunoassay on a Gold-Plated Membrane. Analytical Chemistry, 2013. 85(18): p. 8609-8617.

88. Mosier-Boss, P.A., Review of SERS Substrates for Chemical Sensing. Nanomaterials (Basel), 2017. 7(6).135-142. 
89. Driskell, J.D., R.J. Lipert, M.D. Porter, Labeled Gold Nanoparticles Immobilized at Smooth Metallic Substrates: Systematic Investigation of Surface Plasmon Resonance and Surface-Enhanced Raman Scattering. The Journal of Physical Chemistry B, 2006. 110(35): p. 17444-17451.

90. Fierro-Mercado, P.M. and S.P. Hernández-Rivera, Highly Sensitive Filter Paper Substrate for SERS Trace Explosives Detection. International Journal of Spectroscopy, 2012. 2012: p. $2-7$.

91. Mao, X. T.J. Huang, Microfluidic diagnostics for the developing world. Lab Chip, 2012. 12(8): p. 1412-6.

92. Hu, J., S. Wang, L. Wang, F. Li, B. Pingguan-Murphy, T.J. Lu, and F. Xu, Advances in paper-based point-of-care diagnostics. Biosens Bioelectron, 2014. 54: p. 585-97.

93. Wu, C.C., H.Y. Lin, C.P. Wang, L.F. Lu, T.H. Yu, W.C. Hung, J.Y. Houng, F.M. Chung, Y.J. Lee, and J.J. Hu, Evaluation of a rapid quantitative determination method of PSA concentration with gold immunochromatographic strips. BMC Urol, 2015. 15: p. 109.

94. Parolo, C., A. de la Escosura-Muñiz, and A. Merkoçi, Enhanced lateral flow immunoassay using gold nanoparticles loaded with enzymes. Biosens Bioelectron, 2013. 40(1): p. 412-416.

95. Turkevich, J., P.C. Stevenson, J. Hillier, A study of the nucleation and growth processes in the synthesis of colloidal gold. Discussions of the Faraday Society, 1951. 11(0): p. 5575.

96. Wuithschick, M., A. Birnbaum, S. Witte, M. Sztucki, U. Vainio, N. Pinna, K. Rademann, F. Emmerling, R. Kraehnert, and J. Polte, Turkevich in New Robes: Key Questions Answered for the Most Common Gold Nanoparticle Synthesis. ACS Nano, 2015. 9(7): p. $7052-7071$. 
97. Kim, J.-H., K.M. Twaddle, L.M. Cermak, W. Jang, J. Yun, and H. Byun, Photothermal heating property of gold nanoparticle loaded substrates and their SERS response. Colloids Surf., A, 2016. 498: p. 20-29.

98. Lartey, J.A., J.P. Harms, R. Frimpong, C.C. Mulligan, J.D. Driskell, and J.-H. Kim, Sandwiching analytes with structurally diverse plasmonic nanoparticles on paper substrates for surface enhanced Raman spectroscopy. RSC Adv., 2019. 9. p. 32535 32543.

99. Ngo, Y.H., D. Li, G.P. Simon, and G. Garnier, Gold nanoparticle-paper as a threedimensional surface enhanced raman scattering substrate. Langmuir, 2012. 28(23): p. 8782-8790.

100. Ngo, Y.H., D. Li, G.P. Simon, and G. Garnier, Effect of cationic polyacrylamide dissolution on the adsorption state of gold nanoparticles on paper and their Surface Enhanced Raman Scattering properties. Colloids Surf., A, 2013. 32(4). p. 46-52.

101. Granger, J.H., A. Skuratovsky, M.D. Porter, Courtney L. Scaife, J.E. Shea, Q. Li, and S. Wang, Coupling solid-phase microextractions and surface-enhanced Raman scattering: towards a point-of-need tool for hepatic cancer screening. Analytical Methods, 2017. 9(32): p. 4641-4646.

102. Lopez, A., F. Lovato, S.H. Oh, Y.H. Lai, S. Filbrun, E.A. Driskell, and J.D. Driskell, SERS immunoassay based on the capture and concentration of antigen-assembled gold nanoparticles. Talanta, 2016. 146. p. 388-393. 
103. Wang, G., H.-Y. Park, R.J. Lipert, and M.D. Porter, Mixed Monolayers on Gold Nanoparticle Labels for Multiplexed Surface-Enhanced Raman Scattering Based Immunoassays. Analytical chemistry, 2009. 81(23): p. 9643-9650.

104. Abell, J.L., J.M. Garren, Y. Zhao, Dynamic Rastering Surface-Enhanced Raman Scattering (SERS) Measurements on Silver Nanorod Substrates. Applied Spectroscopy, 2011. 65(7): p. 734-740.

105. de Puig, H., I. Bosch, M. Carré-Camps, and K. Hamad-Schifferli, Effect of theProtein Corona on Antibody-Antigen Binding in Nanoparticle Sandwich Immunoassays. Bioconjugate Chemistry, 2017. 28(1): p. 230-238.

106. Filbrun, S.L. J.D. Driskell, A fluorescence-based method to directly quantify antibodies immobilized on gold nanoparticles. Analyst, 2016. 141(12): p. 3851-3857.

107. Ruiz, G., N. Ryan, K. Rutschke, O. Awotunde, and J.D. Driskell, Antibodies Irreversibly Adsorb to Gold Nanoparticles and Resist Displacement by Common Blood Proteins. Langmuir, 2019. 35(32): p. 10601-10609.

108. Wang, A., K. Vangala, T. Vo, D. Zhang, and N.C. Fitzkee, A Three-Step Model for Protein-Gold Nanoparticle Adsorption. The Journal of Physical Chemistry C, 2014. 118(15): p. 8134-8142.

109. Driskell, J.D., C.G. Larrick, C. Trunell, Effect of Hydration on Plasmonic Couplingof Bioconjugated Gold Nanoparticles Immobilized on a Gold Film Probed by SurfaceEnhanced Raman Spectroscopy. Langmuir, 2014. 30(22): p. 6309-6313.

110. Driskell, J.D., R.J. Lipert, and M.D. Porter, Labeled gold nanoparticles immobilized at smooth metallic substrates: Systematic investigation of surface plasmon resonance and surface-enhanced Raman scattering. Journal of Physical Chemistry B, 2006. 110(35): p. $17444-17451$ 
111. Lee, H.K., Y.H. Lee, C.S.L. Koh, G.C. Phan-Quang, X. Han, C.L. Lay, H.Y.F. Sim, Y.C. Kao, Q. An, and X.Y. Ling, Designing surface-enhanced Raman scattering (SERS) platforms beyond hotspot engineering: emerging opportunities in analyte manipulations and hybrid materials. America Chemical. Society. Review., 2019. 48(3): p. 731-756.

112. Yoon, J.K., K. Kim, and K.S. Shin, Raman scattering of 4-aminobenzenethiol sandwiched between Au nanoparticles and a macroscopically smooth Au substrate: Effect of size of Au nanoparticles. J. Phys. Chem. C, 2009. 113(5): p. 1769-1774.

113. Al-Tamimi, M., W. Shen, R. Zeineddine, H. Tran, and G. Garnier, Validation of PaperBased Assay for Rapid Blood Typing. Analytical chemistry, 2012. 84(3): p. 1661-1668.

114. Wang, J., B. Yiu, J. Obermeyer, C.D.M. Filipe, J.D. Brennan, and R. Pelton, Effects of Temperature and Relative Humidity on the Stability of Paper-Immobilized Antibodies. Biomacromolecules, 2012. 13(2): p. 559-564.

115. Lewis, I.R., N.W. Daniel, P.R. Griffiths, Interpretation of Raman Spectra of NitroContaining Explosive Materials. Part I: Group Frequency and Structural Class Membership. Applied Spectroscopy, 1997. 51(12): p. 1854-1867.

116. Moore, D.S. S.D. McGrane, Comparative infrared and Raman spectroscopy ofenergetic polymers. Journal of Molecular Structure, 2003. 661-662: p. 561-566.

117. Wild, D. and C. Sheehan, Standardization and Calibration, in The immunoassay handbook: theory and applications of ligand binding, ELISA and related techniques, D. Wild, Editor. 2013. 455 - 469. 
118. Penn, M.A., D.M. Drake, J.D. Driskell, Accelerated Surface-Enhanced Raman Spectroscopy (SERS)-Based Immunoassay on a Gold-Plated Membrane. Analytical. Chemistry., 2013. 85: p. 8609-8617.

119. Chen, R., B. Liu, H. Ni, N. Chang, C. Luan, Q. Ge, J. Dong, and X. Zhao, Vertical flow assays based on core-shell SERS nanotags for multiplex prostate cancer biomarker detection. Analyst, 2019. 144(13): p. 4051-4059.

120. Zhang, D., L. Huang, B. Liu, Q. Ge, J. Dong, and X. Zhao, A vertical flow microarray chip based on SERS nanotags for rapid and ultrasensitive quantification of $\alpha$ fetoprotein and carcinoembryonic antigen. Microchimica Acta, 2019. 186(11): p. 664699. 